\title{
Influence de l'extraction des granulats en mer sur l'équilibre du littoral
}

\section{Influence of offshore coarse-grained material extraction on coastal equilibrium}

\author{
Claude Migniot et Jacques Viguier \\ Département Sédimentologie \\ Laboratoire Central d'Hydraulique de France *
}

\section{Résumé}

L'extraction en mer de sables ou de graviers nécessite de respecter des conditions de dragage bien précises si l'on veut éviter que ces extractions aient une influence néfaste sur le littoral.

Des études théoriques et des mesures en nature ont permis de préciser l'importance des mouvements sédimentaires à différentes distances de la côte sous l'action des houles. Dans le cas du Golfe de Gascogne, où les houles sont particulièrement fortes, on constate qu'il faut dépasser les profondeurs de $20 \mathrm{~m}$ sous le niveau des plus basses mers pour que les déplacements saisonniers de sable soient négligeables sur des fonds réguliers en pente douce.

Des études en canal vitré et en cuve à houle ont apporté des renseignements sur l'évolution des fosses d'emprunt de sables dragués à différentes profondeurs entre le littoral et les fonds de $-25 \mathrm{~m}$. Ces fosses avaient une profondeur de $6 \mathrm{~m}$ sous le niveau du fond marin et une largeur au sommet de $200 \mathrm{~m}$.

Les résultats obtenus montrent que de telles fosses commencent à se combler à partir d'une amplitude critique de la houle $(H c)$ égale à 0,28 fois la profondeur df au point considéré

$$
H c=0,25 \text { à } 0,30 d f
$$

Le taux de comblement de ces fosses de dragages peut être déterminé à partir de l'amplitude de la houle $H_{1 / 10}$ par l'expression :

$$
V \text { en } \mathrm{m}^{3} / \mathrm{m} / \text { jour }=\left(H_{1 / 10}-H c\right)^{1,5}
$$

Les dépôts se forment principalement par adoucissement de la pente du talus côté terre et entraînent des

(*) L.C.H.F. 10, rue liugène Renault. 94700 MAISONS ALFORT. érosions vers les petits fonds et, par voie de conséquence, vers la plage.

Si la fosse de dragage est située au-delà des fonds de $-21 \mathrm{~m}$ sous le niveau des basses-mers, son action sur les fonds avoisinants et le littoral est pratiquement inexistante tant que l'amplitude maximale des houles ne dépasse pas $9 \mathrm{~m}$.

Les résultats de cette étude permettront de prévoir les conditions de colmatage de fosses de dragage en mer et de fixer les profondeurs limites d'extraction des agrégats en fonction des conditions océanographiques locales pour que les répercussions sur le littoral soient négligeables.

\section{Introduction}

L'extraction d'agrégats en bordure de mer entraîne fréquemment des perturbations dans l'équilibre du littoral se traduisant par des érosions importantes et une dégradation progressive de nos côtes.

Il suffit de rappeler les reculs spectaculaires du trait de côte de plus de 15 mètres par an dans des secteurs où des emprunts de sables, destinés à la construction ou imposés par des améliorations portuaires, avaient été effectués, pour appeler à une extrême prudence dès qu'il s'agit d'apporter une perturbation à un équilibre du littoral déjà très précaire.

La solution de facilité consiste à interdire purement et simplement de telles extractions comme on tend de plus en plus à le faire en rivière. C'est méconnaître les besoins sans cesse croissants en sables, graviers ou galets qui dépassent actuellement en France 250 millions de tonnes par an pour la partie extraite par dragages.

Sans vouloir prétendre que le sous-sol marin du plateau continental contient une réserve inépuisable d'agrégats on peut penser qu'une exploitation ration-

LA HOUILLE BLANCHE 
nelle des sédiments situés dans les petits fonds marins, à une certaine distance de la côte apporterait une solution partielle pour la fourniture d'agrégats.

C'est dans ce sens que le Centre National pour l'Exploitation des Océans (C.N.E.X.O) a entrepris un inventaire des ressources sédimentaires disponibles le long du plateau continental et que, parallèlement, le Laboratoire Central d'Hydraulique de France (L.C.H.F.) a effectué, en association avec la Section d'Application de la Radioactivité (S.A.R.) du Centre d'Etudes Nucléaires de Saclay, une première série de recherches en vue d'essayer de mieux quantitifier les mouvements de sédiments situés à différentes profondeurs.

Devant l'intérêt de ces recherches et compte tenu de la masse sédimentaire disponible en mer, qui atteint 55 milliards de tonnes, la Direction Départementale des Pyrénées Atlantiques et le Service Central Technique de la Direction des Ports et Voies Navigables (S.C.T.) se sont associés à ces études en confiant au L.C.H.F. des recherches en modèle réduit permettant de fixer l'influence des souilles d'emprunts d'agrégats réalisées entre les fonds de -5 et de $-25 \mathrm{~m}$ sous le niveau des plus basses-mers (fig. 0 ).

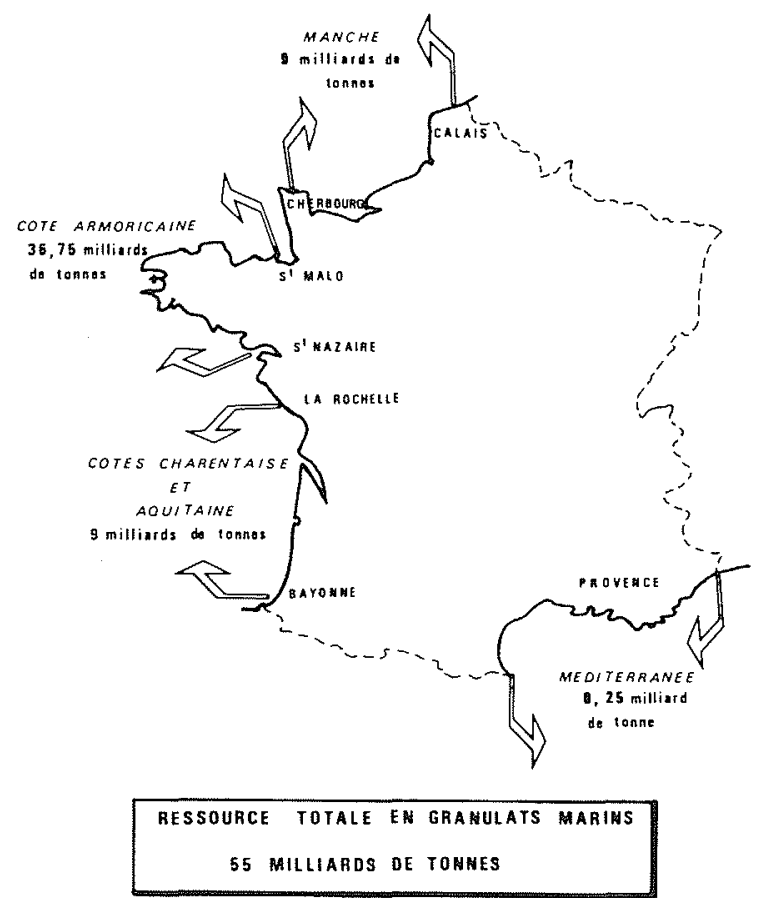

Figure 0 - (D'après A. BRIN - documents CNEXO).

Les résultats obtenus sont regroupés dans cette synthèse et apportent un certain nombre d'éléments permettant de préciser l'influence d'extraction de sables et de graviers en mer sur la tenue du littoral et de fixer la profondeur limite à partir de laquelle ces extractions pourraient être autorisées.
Etude des mouvements sédimentaires littoraux sous l'action des houles à différentes profondeurs

\section{Généralités}

Sous l'action des houles les sédiments peuvent être soumis à des mouvements très divers allant de la simple oscillation sur les fonds, sous l'effet des mouvements orbitaires, à des déplacements dans le profil perpendiculairement à la côte, ou à une dérive le long du littoral sous l'action des houles obliques (fig. 1, 2, 3).

Les caractéristiques de la houle, la nature des sédiments, la morphologie des fonds et du littoral vont intervenir dans les phénomènes de transport, l'équilibre sédimentaire final n'étant qu'une résultante d'actions hydrodynamiques où fluides et solides réagissent l'un sur l'autre.

Les lois qui régissent ces phénomènes restent encore imparfaitement connues mais il est possible en associant les données théoriques à des études en nature basées sur les évolutions des fonds, les triages granulométriques et les expériences à l'aide de traceurs radioactifs et fluorescents, d'estimer l'importance des mouvements sédimentaires à différentes profondeurs du littoral.

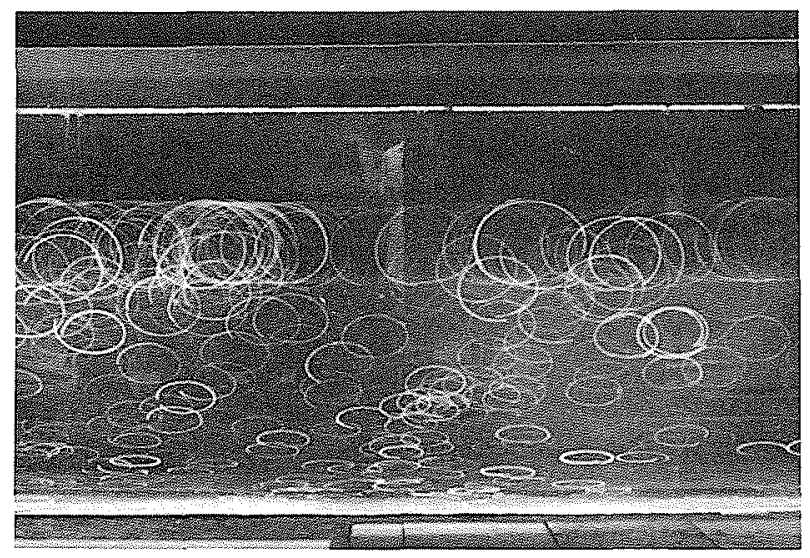

Figure 1 - Mouvements orbitaires.

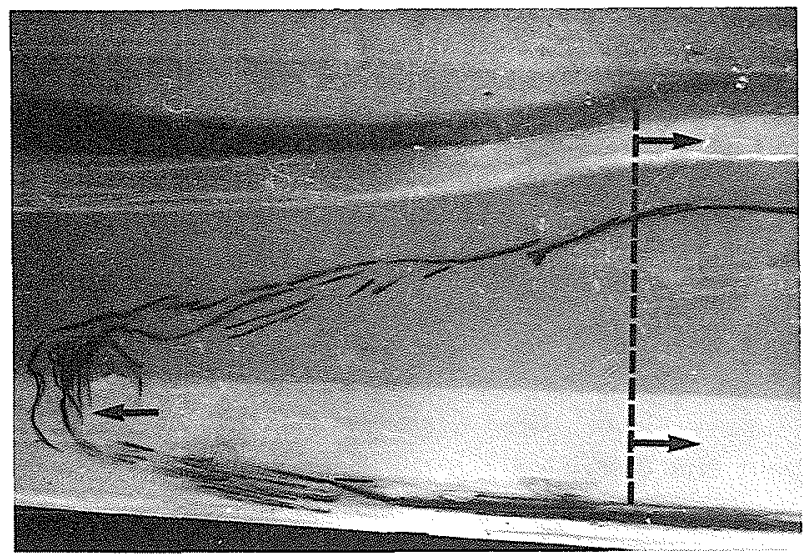

Figure 2 - Mouvements des masses d'eau sous l'action de la houle. 


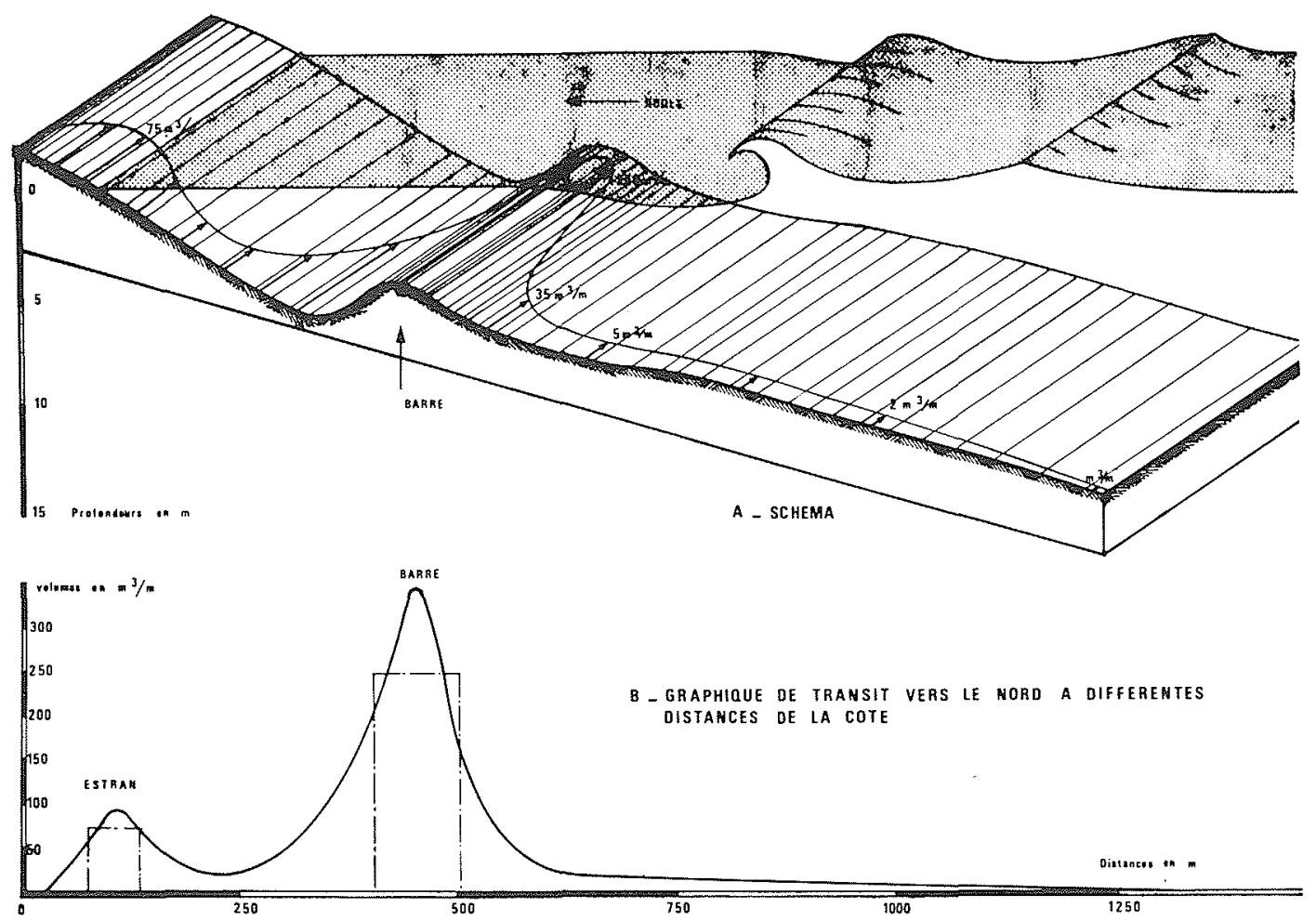

Figure 3 - Répartition des transports le long d'un profil

Les études effectuées par le L.C.H.F. et d'autres organismes de recherches dans les différentes régions du monde, au cours des trente dernières années, ont montré que les mouvements dans le profil étaient importants entre la côte et les fonds de -7 à $-10 \mathrm{~m}$. Dans cette zone, les fonds s'érodent et se sédimentent alternativement, l'évolution résultante dépendant des caractéristiques des houles.

Entre les fonds de $-10 \mathrm{~m}$ et de $-15 \mathrm{~m}$ à $-20 \mathrm{~m}$ des variations plus faibles des profondeurs peuvent être observées, les matériaux étant soit emportés par les courants de compensation vers le large, soit ramenés vers la côte au début de la période de beau temps. La résultante de ces deux mouvements se traduit, à chaque tempête, par une "perte" en matériaux fins qui sont entraînés vers les plus grandes profondeurs où ils ne peuvent être repris.

Dans tous les cas il ne semble pas que des remaniements sédimentaires de grande importance puissent être observés au-delà des fonds de $-20 \mathrm{~m}$ et, dans la plupart des cas, les vases apparaissent à partir des fonds de -30 à $-40 \mathrm{~m}$ éliminant une possibilité d'alimentation en sables des zones côtières par les sédiments provenant des plus grandes profondeurs.

Les mesures en nature montrent que les remaniements annuels dans le profil peuvent atteindre $500000 \mathrm{~m}^{3}$ par kilomètre de côte et se localisent principalement entre les fonds de $-10 \mathrm{~m}$ et l'estran. En absence d'ouvrages ou d'emprunt de sable le long du littoral, le profil d'équilibre moyen des fonds peut être considéré comme stable au cours d'une année.

A ces mouvements dans le profil se superpose en général un déplacement parallèle à la côte sous l'action des houles obliques ou sous l'action des courants généraux.

Ce transport littoral est très variable et peut atteindre 2 millions de $\mathrm{m}^{3}$ par an au maximum ou seulement quelques milliers de $\mathrm{m}^{3}$ par an. Il s'effectue entre le déferlement et la côte et devient faible à négligeable audelà du point de déferlement de la houle.

Si par suite de réalisation d'ouvrages ou d'emprunts de matériaux dans la zone "active" du courant de houle on introduit un déséquilibre dans les phénomènes de transport, on entraînera automatiquement une érosion des petits fonds marins et, par voie de conséquence, un recul du littoral.

Etude théorique de la limite d'action des houles sur les fonds

Les théories sur la houle permettent d'effectuer une première approche des mouvements des sédiments à différentes profondeurs, pour des houles de diverses amplitudes et périodes et pour différents types de matériaux.

Sous l'action des mouvements orbitaires de la houle, les fonds marins peuvent être soumis à des vitesses alternatives susceptibles de provoquer des mouvements de sédiments si ces vitesses dépassent un certain seuil critique propre à chaque type de matériau.

La théorie montre que l'on peut déterminer la vitesse de frottement $u_{*}$ sur le fond par l'expression :

$$
u_{*}=\left(\frac{8 \nu U^{2} \max }{\pi T}\right)^{1 / 4}
$$


ou dans une eau à $20^{\circ} \mathrm{C}$ :

dans laquelle :

$$
u_{*}=0,04 \frac{U_{\max }^{1 / 2}}{T^{1 / 4}}
$$

$\nu \quad$ est la viscosité cinématique de l'eau

$$
\left(10^{-6} \mathrm{~m}^{2} / \mathrm{s} \text { à } 20^{\circ} \mathrm{C}\right)
$$

$U_{\max }$ est la vitesse orbitaire maximale sur le fond

$T^{\max } \quad$ est la période de la houle

ce qui donne, dans le système S.I. en tenant compte de la valeur de $U_{\max }$ :

$$
u_{*}=0,07\left(\frac{H^{2}}{T^{3} \operatorname{sh}^{2} 2 \pi \frac{d}{L}}\right)^{1 / 4}
$$

dans laquelle :

$H \quad$ est l'amplitude de la houle (hauteur entre creux et crête de la vague)

$L \quad$ est la longueur d'onde

d est la profondeur d'eau.

Connaissant la vitesse théorique de frottement $u_{*}$ sur les fonds ou la force tractrice $\tau_{0}$ on peut, par analogie avec les mesures faites sur le début d'entraînement des particules de sable par les courants, prévoir la limite d'action des houles sur des sables de diamètre donné.

D'une façon simplifiée on peut admettre, avec une assez bonne approximation, que la force tractrice critique $\tau_{c}$ nécessaire pour provoquer le début d'entraînement de particules d'un diamètre $D$ (en $\mathrm{m}$ ) est donnée par l'expression :

$$
\tau_{c}\left(\text { en } N / \mathrm{m}^{2}\right)=50\left(\rho_{s}-\rho_{0}\right) D g=u_{*}^{2} \rho
$$

$\rho_{s}$ et $\rho_{0}$ étant la masse spécifique des sédiments et de l'eau (en $\mathrm{kg} / \mathrm{m}^{3}$ ), $g$ l'accélération de la pesanteur (en $\mathrm{m} / \mathrm{s}$ ).

La figure 4 donne la vitesse critique en fonction du diamètre des particules.

En égalant cette équation et celle de la force tractrice $\tau_{0}$ exercée par la houle sur les fonds on peut avoir une première idée de la limite du début d'oscillation des particules sur les fonds.

A titre d'exemple en appliquant ces différentes formules aux houles du Golfe de Gascogne, qui peuvent être considérées comme extrêmement fortes (fig. 5), on peut tracer les valeurs de $u_{*}$ en fonction des amplitudes pour différentes profondeurs des fonds et en déduire les limites extrêmes des mouvements sédimentaires des particules plus ou moins grosses (fig. 6). Rappelons que dans le Golfe de Gascogne il existe une relation entre les amplitudes et les périodes de la forme $T=0,8$ à $1,4 H+7,5$.

D'après cette approche théorique on constate que : - Par des profondeurs de $-10 \mathrm{~m}$ sous le niveau de l'eau, les particules supérieures à $1,6 \mathrm{~mm}$ ne pourront être déplacées quelle que soit l'amplitude des houles. Par contre, à partir d'une amplitude de $5 \mathrm{~m}$, les particules de $1 \mathrm{~mm}$ seront mises en mouvement et il suffira d'une houle de $2 \mathrm{~m}$ pour provoquer le début d'oscillation des particules fines comprises entre 0,4 et $0,2 \mathrm{~mm}$.

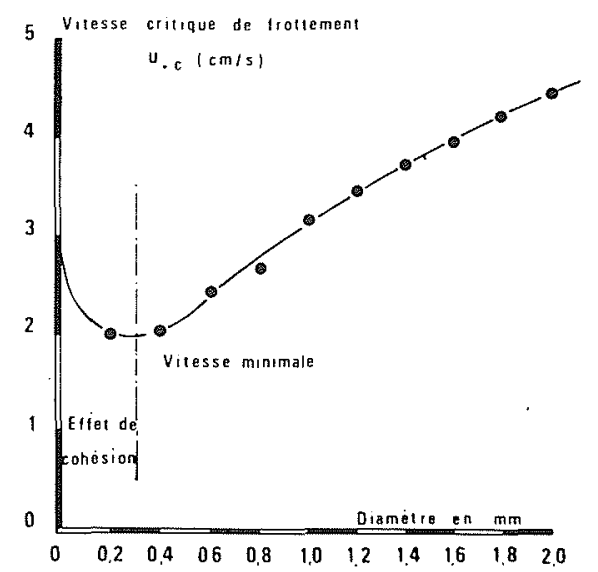

Figure 4 - Variation de la vitesse critique de frottement nécessaire pour provoquer le début d'entraînement des sables.

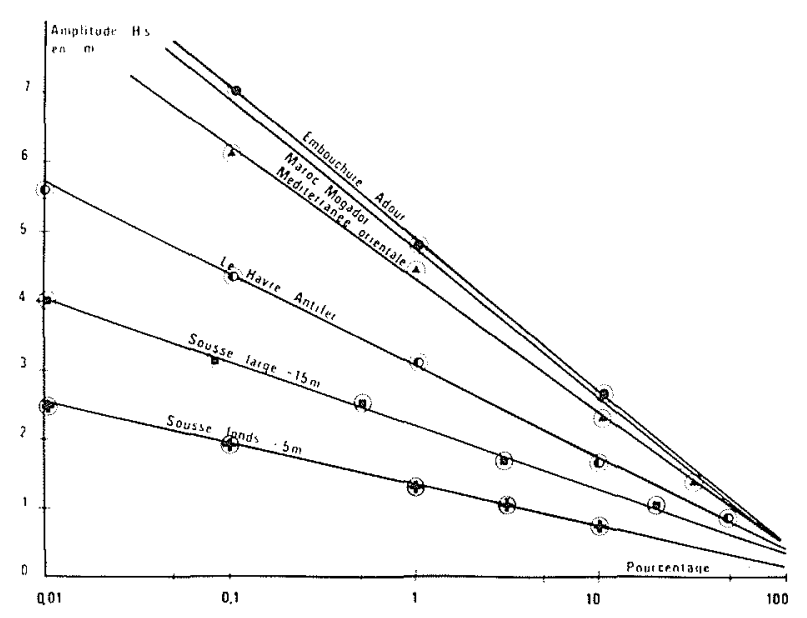

Figure 5 - Répartition des amplitudes significatives dans différents ports.

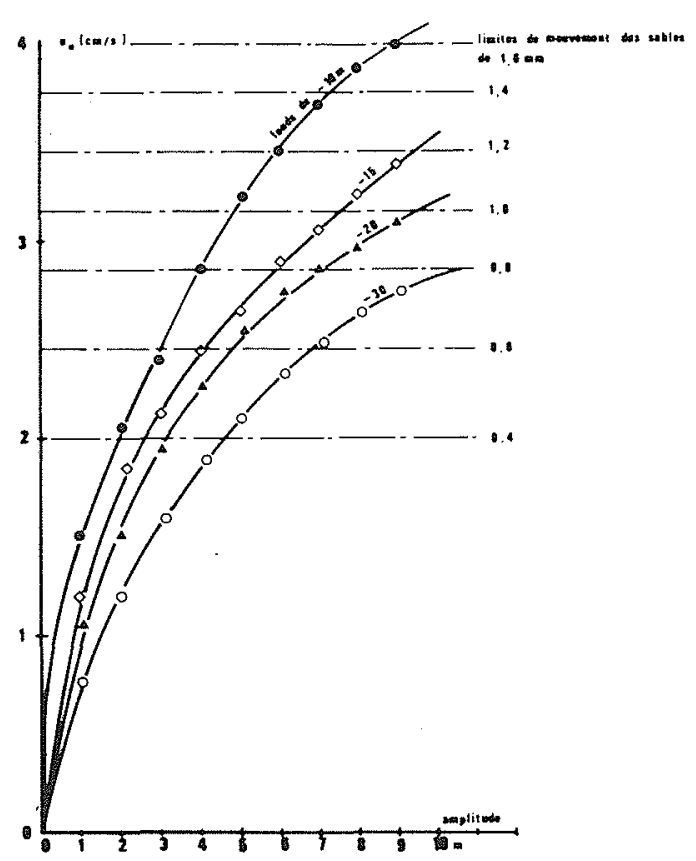

Figure 6 -- Variation de la vitesse de frottement $u_{*}$ en fonction de l'amplitude de la houle. 
- Par des profondeurs de $-20 \mathrm{~m}$ les particules supé rieures à $1 \mathrm{~mm}$ ne se déplaceront pas quelles que soient les houles et il faudra que l'amplitude dépasse $5 \mathrm{~m}$ pour déplacer des grains de $0,6 \mathrm{~mm}$. Par contre, les sables fins de 0,4 et $0,2 \mathrm{~mm}$ subiront un début d'oscillation à partir d'une amplitude de 3 à $3,50 \mathrm{~m}$ (10\% du temps).

- Par des profondeurs de $--30 \mathrm{~m}$ il faudra que les houles dépassent $4,50 \mathrm{~m}$ pour provoquer le début d'oscillation des sables fins, les sables plus grossiers ou ayant une cohésion propre (sables de $0,10 \mathrm{~mm}$ et inférieurs) restant pratiquement stables.

\section{Importance des mouvements sédimentaires et déplace- ment théorique des materiaux}

Le fait que des particules sableuses fines puissent subir un début d'entraînement sous l'effet des vitesses oscillatoires dues à la houle ne signifie pas que les mouvements sédimentaires soient appréciables. Les sables peuvent très bien subir une oscillation sur place sans qu'il $y$ ait déplacement dans un sens préférentiel.

Pour qu'il y ait déplacement des sédiments ayant subi un début de mise en oscillation il faut en plus qu'un courant se superpose au phénomène. De tels courants peuvent être engendrés :

- soit par les courants généraux dus à la marée, ils sont en général très faibles le long du littoral Sud des Landes $(0,10$ à $0,15 \mathrm{~m} / \mathrm{s})$,

soit par les courants prenant naissance sous l'effet de la houle au voisinage du fond.

Danș ce cas les sédiments pourront être entraînés vers la côte si la couche est laminaire ou dispersés verticalement dans la tranche d'eau si la couche limite est turbulente et être entrainés vers le large par le courant de masse (fig. 2). Par des profondeurs de 20 à $30 \mathrm{~m}$, les courants de translation dans la couche limite ne dépasseraient pas quelques centimètres par seconde.

On peut également présumer, par la théorie, que les mouvements sédimentaires sous l'action des houles seront faibles par des fonds de $-20 \mathrm{~m}$ à $-30 \mathrm{~m}$ en effectuant un calcul des quantités mises en mouvement par oscillation. L'application des lois d'Einstein ou de Meyer Peter, donnant le débit solide transporté " $g s$ " par les courants, permet d'approcher ce problème en tenant compte, par ailleurs, du nombre de jours d'action des houles ayant une amplitude déterminée le long de la côte.

Il faut bien préciser que par ce calcul on évalue la masse totale du sédiment qui oscille sur les fonds mais non son déplacement dans un sens préférentiel ; en fait il ne pourra partir qu'une certaine quantité de ces matériaux mis en mouvement "sur place".

Toujours en prenant pour exemple le Golfe de Gascogne et en tenant compte de la probabilité de chaque type de houle dans l'année, on obtient le graphique des remaniements théoriques à différentes profondeurs en fonction de l'amplitude de la houle, donné par la figure 7.

Ce graphique montre d'une part l'amplitude de houle qui remanie la plus grosse quantité de matériau à une profondeur donnée (par exemple par fonds de $-10 \mathrm{~m}$ c'est la houle de $3,50 \mathrm{~m}$ qui transporte le plus de maté-

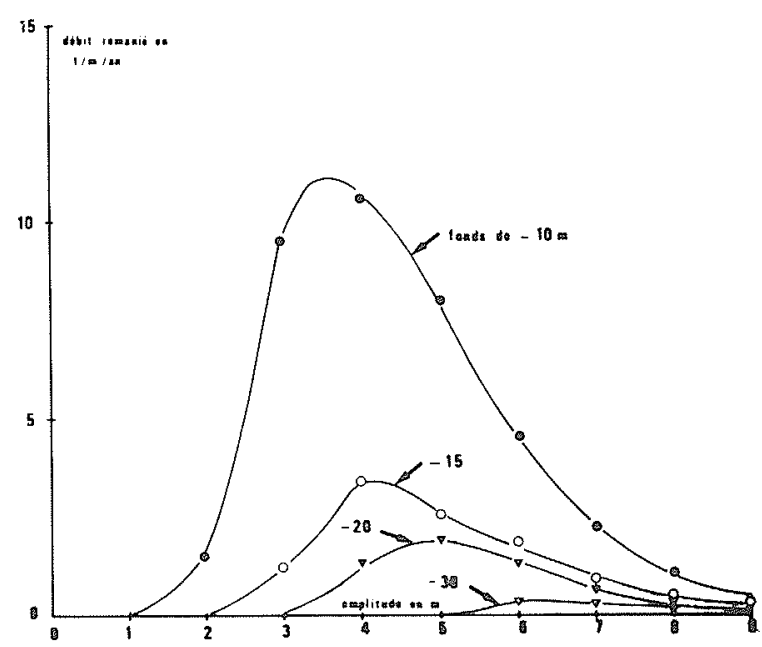

Figure 7 - Graphique des remaniements théoriques à différentes profondeurs en fonction de l'amplitude de la houle.

riau dans le profil et par fonds de $-20 \mathrm{~m}$ la houle de $5 \mathrm{~m}$ ), d'autre part les valeurs relatives des quantités remaniées au cours de l'année en fonction des profondeurs.

Pour un bilan annuel total $\Sigma H^{2} T t$ ( $t$ étant la durée d'action d'une houle d'amplitude $H$ et de période $T$ ) de $2,1 \times 10^{9} \mathrm{~m}^{2} \mathrm{~s}^{2}$, on obtient à différentes profondeurs les volumes théoriques remaniés suivants par application soit des lois d'Einstein soit de Meyer Peter (fig. 8).

\begin{tabular}{|c|c|c|c|c|c|}
\hline \multicolumn{6}{|c|}{$\begin{array}{c}\text { Comparaison des volumes theor iques remanies par la houle } \\
\text { d'après des formules type heyer Peter et Einstein }\end{array}$} \\
\hline Profondeur (en m) & 7 & 10 & 15 & 20 & 30 \\
\hline $\begin{array}{c}\text { Volunits renanies en m }{ }^{3} / \mathrm{m} / \mathrm{an} \\
\text { (heyer Peter) }\end{array}$ & 385 & 38 & 13 & 5,5 & 0,5 \\
\hline (Einstein) & 180 & 72 & 19 & 10 & 2 \\
\hline
\end{tabular}

Sans vouloir attacher une valeur quantitative et absolue aux résultats de ces calculs, on constate que :

- les quantités de sable fin de 200 à 300 microns susceptibles d'être remaniées par l'action des houles diminuent très rapidement avec la profondeur ; elles sont encore importantes par fonds de $-10 \mathrm{~m}$ et pratiquement nulles par fonds de $-30 \mathrm{~m}$,

- on peut estimer que par fonds de $-30 \mathrm{~m}$, les risques de remaniement des fonds sont en moyenne 50 fois plus faibles que par fonds de $-10 \mathrm{~m}$,

- par fonds de $-15 \mathrm{~m}$, par contre, les remaniements restent appréciables,

- plus on se rapproche du littoral, plus les houles de faible amplitude interviennent dans le remaniement global des fonds.

Sur le plan théorique, on ne peut donc que conseiller de limiter les extractions de sable à des profondeurs supérieures à $-20 \mathrm{~m}$. En prenant des limites situées entre -25 et -30 , on se trouve dans le cas du Golfe de Gascogne dans des profondeurs où les risques de remaniement sont très faibles. Pour des houles du type de la Méditerranée ou dans la Manche, il est probable qu'une approche théorique du problème montrerait que 


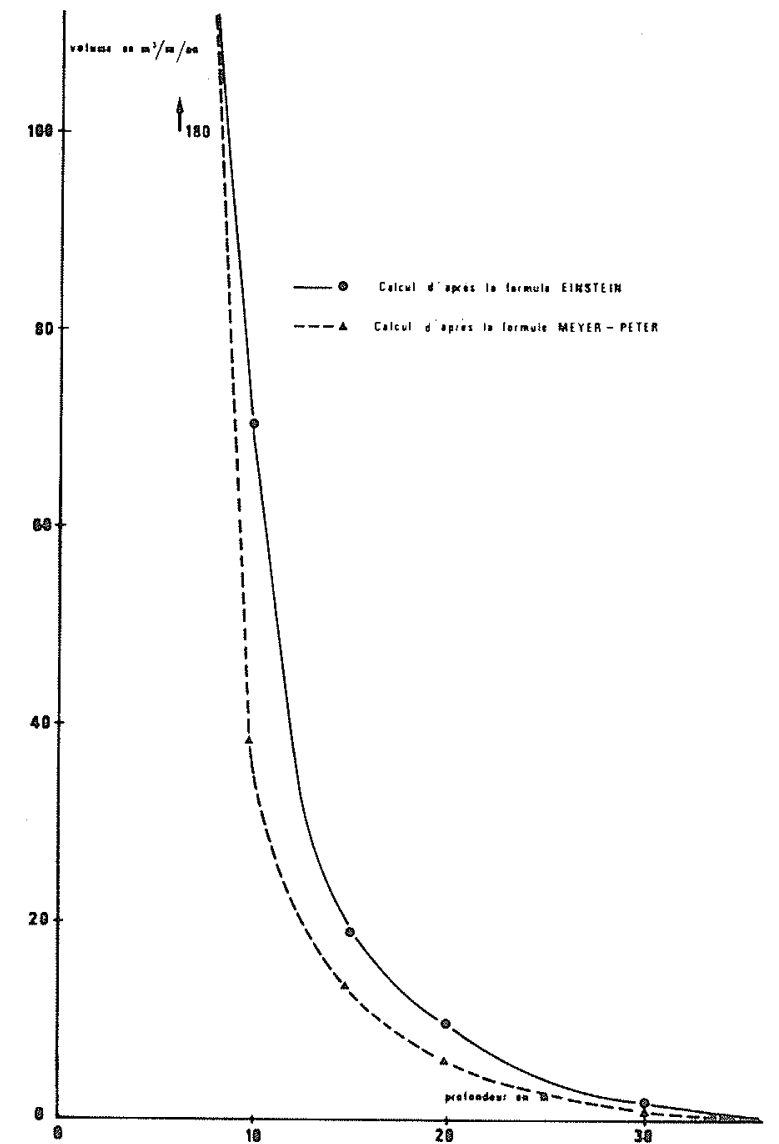

Figure 8 - Volume théorique des sédiments soumis à un mouvement alternatif.

l'on peut extraire des sables dans des profondeurs sensiblement plus faibles.

\section{Mesures en nature des mouvements des sédiments sous la houle à l'aide des traceurs radioactifs}

Pour quantifier en nature l'importance des mouvements sédimentaires sous l'action de la houle, différentes mesures ont été réalisées en nature par le L.C.H.F. en association avec la Section d'Application de la Radioactivité (S.A.R.) du Centre d'Etudes Nucléaires de Saclay. Ces mesures ont été complétées par des expériences faites en Angleterre et au Brésil.

Il ressort de cette étude que :

- des galets ou graviers de 2 à $4 \mathrm{~cm}$ de diamètre ne bougent pas par des fonds de $-18 \mathrm{~m}$ en Manche (expérience Angleterre). Par fonds de $-12 \mathrm{~m}$ ils remonteraient vers la côte avec un débit de $0,5 \mathrm{~m}^{3}$ par an et par mètre linéaire de plage et de $1,5 \mathrm{~m}^{3} / \mathrm{m} /$ an par fonds de $-9 \mathrm{~m}$. De tels débits représentent des mouvements extrêmement faibles (200 ans pour qu'un galet passe des fonds de $-12 \mathrm{~m}$ à des fonds de $-9 \mathrm{~m}$ ),

des particules de 2 à $3 \mathrm{~mm}$ de sable grossier ne sont pas remaniées par des houles de 6 à $7 \mathrm{~m}$ d'amplitude lorsque ces particules sont déposées par des profondeurs supérieures à $-14 \mathrm{~m}$ sous le niveau de l'eau. Les mouvements apparaissent par des profondeurs de $-10 \mathrm{~m}$ et l'entraînement est total par des fonds de $-7 \mathrm{~m}$. une partie des gros éléments étant remontée à la côte (expériences L.C.H.F. S.A.R. faites devant la côte d'Anglet),

- des sables de 0,3 mm déposés par des fonds de $-14 \mathrm{~m}$ ne bougeraient pratiquement pas pour des houles de 3 à $4 \mathrm{~m}$ d'amplitude (Commissariat à l'Energie Atomique Brésilien - 1973),

- des sables de 0,2 à $0,4 \mathrm{~mm}$ immergés par des fonds de $--7 \mathrm{~m}$ sont remontés à la côte par des houles de $2,50 \mathrm{~m}$ d'amplitude. Par contre par fonds de $-12 \mathrm{~m}(-10 \mathrm{~m}$ sous zéro $\mathrm{CM})$ les mêmes sables ne bougeraient pratiquement pas pour des houles atteignant $5 \mathrm{~m}$ d'amplitude (expériences L.C.H.F. S.A.R. côte d'Anglet et Méditerranée Orientale).

On constate également au cours de ces expériences que l'épaisseur de la couche mobile de sable remaniée par les houles, ne dépasserait pas 0,5 à $1 \mathrm{~cm}$ par fonds de $-22 \mathrm{~m}, 2 \mathrm{~cm}$ par fonds de $-15 \mathrm{~m}, 16$ à $27 \mathrm{~cm}$ par fonds de $-8 \mathrm{~m}, 50 \mathrm{~cm}$ par fonds de $-6 \mathrm{~m}$ et seulement $12 \mathrm{~cm}$ à l'estran. Ces épaisseurs dépendent évidemment de la nature des matériaux et des caractéristiques des houles, les valeurs indiquées se rapportant au Golfe de Gascogne et ne tenant pas compte des baïnes ou des barres.

Des expériences récentes effectuées entre le 15 Septembre et le 15 Décembre 1975 par la S.A.R. pour le compte du Service Central Technique de la Direction des Ports et des Voies Navigables permettent de quantifier l'importance des volumes sédimentaires déplacés par les houles à différentes profondeurs dans le Golfe de Gascogne, au Nord de l'embouchure de l'Adour. Au cours de cette période l'obliquité des houles avec la côte était très faible $\left(0\right.$ à $5^{\circ}$ avec une résultante vers le Sud).

Pour un bilan d'énergie de $42010^{6} \mathrm{~m}^{2} \mathrm{~s}^{2}$ représentant le cinquième du bilan d'énergie annuelle au même point les valeurs suivantes des mouvements des sables vers la côte ont été mesurées :

\begin{tabular}{|c|c|c|c|c|c|}
\hline $\begin{array}{c}\text { Profondeur } \\
\text { (en nis sous basse-ner) }\end{array}$ & -6 & -8 & $-15,2$ & $-15,5$ & $-22,2$ \\
\hline $\begin{array}{l}\text { Diamètre des grains } \\
\text { (en microns) }\end{array}$ & $\begin{array}{l}500 \\
\text { à } \\
800\end{array}$ & $\begin{array}{c}160 \\
\bar{a} \\
240\end{array}$ & $\begin{array}{c}125 \\
\dot{a} \\
200\end{array}$ & $\begin{array}{c}320 \\
\dot{a} \\
500\end{array}$ & $\begin{array}{c}100 \\
\bar{a} \\
160\end{array}$ \\
\hline $\begin{array}{l}\text { Vol une transporté } \\
\text { en } 3 \text { mo is }\left(\mathrm{m}^{3} / \mathrm{m}\right)\end{array}$ & 85 & 83 & 1,3 & 11,9 & 0,4 \\
\hline $\begin{array}{l}\text { Volunie annuel } \\
\text { en } \mathrm{m}^{3} / \mathrm{m} / \mathrm{an}\end{array}$ & 425 & 415 & 6,5 & 59,5 & 2 \\
\hline
\end{tabular}

Le volume transporté en $\mathrm{m}^{3} / \mathrm{m}$ à différentes profondeurs $d$ serait donné par l'expression :

$$
V \mathrm{~m}^{3} / \mathrm{m}=f(d) \Sigma H^{2} T t
$$

Par fonds de $-8 \mathrm{~m}$, la valeur de $f(d)$ serait de $210^{-7} \mathrm{~s}^{-2}$

Par fonds de $-10 \mathrm{~m}$, elle ne serait plus que de $210^{-8} \mathrm{~s}^{-2}$ et tomberait à $0,7510^{-8} \mathrm{~s}^{-2}$ par fonds de $-15 \mathrm{~m}$.

Ces valeurs du coefficient $f(d)$ doivent être modulées pour tenir compte de la profondeur du déferlement. Pour des tempêtes de $7 \mathrm{~m}$ d'amplitude, susceptibles de 


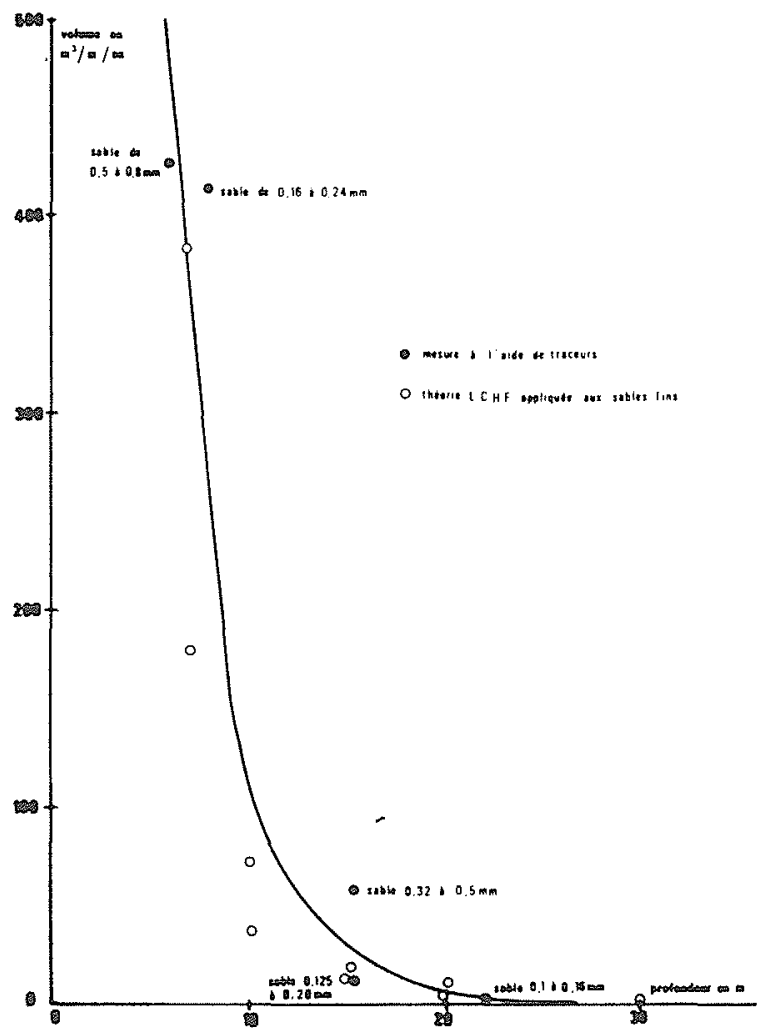

Figure 9 - Volume des sédiments sableux déplacés dans le profil en fonction de la profondeur. (Résultats des expériences à l'aide de traceurs radioactifs de 1975 ramenés à une année moyenne).

déferler par des fonds de $9 \mathrm{~m}$ la valeur de $f(d)$ sera plus importante pour cette profondeur que pour des profondeurs plus faibles, où la houle a perdu une partie de son énergie.

Si l'on extrapole l'ensemble des résultats obtenus à une année moyenne, on constate que les quantités tranportées par fonds de $-8 \mathrm{~m}$, dans le profil, perpendiculairement à la côte, seraient de :

$$
\begin{aligned}
V \mathrm{~m}^{3} / \mathrm{m} / \mathrm{an}=210^{-7} \mathrm{~s}^{-2} \cdot 2,110^{9} \mathrm{~m}^{2} \mathrm{~s}^{2}= \\
420 \mathrm{~m}^{3} / \mathrm{m} / \mathrm{an}
\end{aligned}
$$

valeur très voisine de celle que le L.C.H.F. avait indiquée à partir de l'étude de l'évolution des profils (500000 $\mathrm{m}^{3} /$ an/kilomètre de plage).

De même lorsque l'on compare les résultats des études en nature à l'aide de traceurs aux prévisions théoriques faites antérieurement (voir plus haut) on trouve des valeurs du même ordre de grandeur (fig. 9).

\section{Conclusion de l'étude des mouvements sédimentaires à différentes profondeurs du plateau continental}

Les constatations que l'on peut faire soit en étudiant directement en nature l'évolution des fonds et les variations granulométriques des sédiments au cours du temps, soit en essayant d'aborder le problème par la théorie, soit enfin en interprétant les différents résultats obtenus à l'aide de traceurs radioactifs disposés sur les fonds marins, conduisent toutes à montrer que les déplacements des sédiments sous l'action des houles frontales, inférieures à $7 \mathrm{~m}$ d'amplitude, sont pratique- ment nuls pour des profondeurs de $-30 \mathrm{~m}$ et très faibles pour des fonds de $-20 \mathrm{~m}$.

A partir des fonds de $-15 \mathrm{~m}$ par contre, les mouvements sédimentaires dans le profil deviennent appréciables et augmentent très vite lorsque l'on se rapproche de la côte pour atteindre 400 à $500000 \mathrm{~m}^{3}$ par kilomètre de plage dans les fonds de -7 à $-8 \mathrm{~m}$ dans le cas du Golfe de Gascogne et de la Méditerranée Orientale.

Ces valeurs ne tiennent pas compte des transports sédimentaires sous l'action des houles obliques qui forment le long du littoral un véritable "fleuve côtier" capable d'entraîner des masses de matériaux sur de longues distances parallèlement au rivage et des dispersions de ces mêmes matériaux vers le large sous l'action des rip-currents.

Ces résultats sont applicables à des fonds réguliers en pente douce et leur extrapolation à des fosses de dragages importantes doit être faite avec réserve. Il semble toutefois qu'en limitant les dragages en mer à des profondeurs comprises entre -20 et $-30 \mathrm{~m}$, sous le zéro des plus basses mers, on se situe dans un secteur où les mouvements sédimentaires sont très faibles, évitant ainsi des dégradations appréciables de la côte.

Influence des souilles d'emprunt d'agrégats réalisées entre les fonds de -5 et $-25 \mathrm{~m}$

\section{Généralités - Conditions expérimentales}

Pour mieux cerner le problème de l'influence de souilles d'emprunt de sédiments sur l'équilibre des fonds, et en particulier sur l'équilibre du littoral, une première série d'études en modèle réduit a été entreprise au L.C.H.F. en examinant le comportement de fosses de dragages réalisées à des profondeurs croissantes entre les fonds de $-6 \mathrm{~m}$ et de $-23 \mathrm{~m}$.

Ces études ont été faites en choisissant des échelles de similitude qui permettent de représenter correctement les mouvements des sédiments sous l'action de la houle et en contrôlant à partir des mesures faites en nature que les déplacements des matériaux artificiels sur le modèle sont conformes aux déplacements des sables mesurés en nature pour des conditions hydrodynamiques déterminées. Une échelle en plan de 1/200 et une échelle des hauteurs de $1 / 75$ ont été adoptées.

Des recherches en canal vitré (fig. 10) ont tout d'abord permis de définir l'amplitude critique de la houle susceptible d'entrainer un début de comblement de la souille en fonction de sa profondeur. Les conditions de colmatage ont ensuite été analysées ainsi que les différentes formes prises par les dépôts.

Cette étude en canal vitré, à deux dimensions, a été complétée par des recherches beaucoup plus poussées en cuve à houle à trois dimensions permettant de prendre en compte l'ensemble des phénomènes hydrody. namiques qui interviennent dans les mouvements sédimentaires (fig. 11).

Dans tous les cas les houles reproduites se rapprochaient des houles naturelles, leur succession dans le temps s'effectuant sous forme de trains d'ondes dont la 


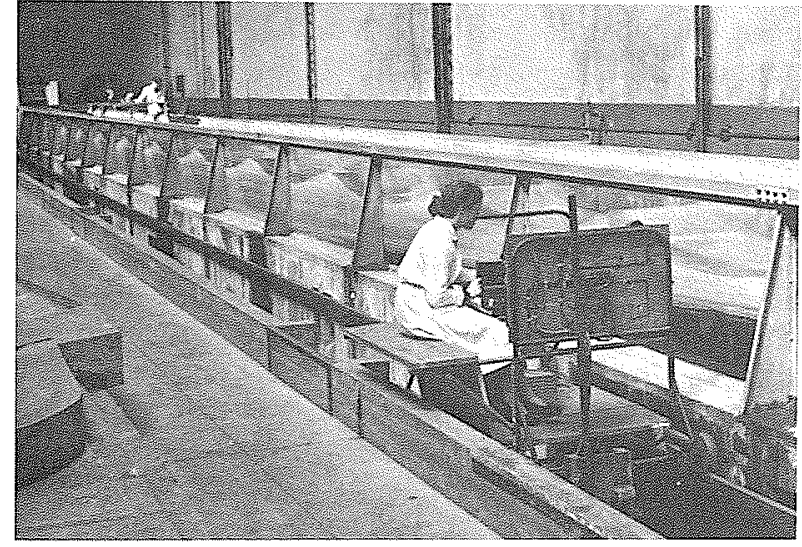

Figure 10 - Canal à houle.

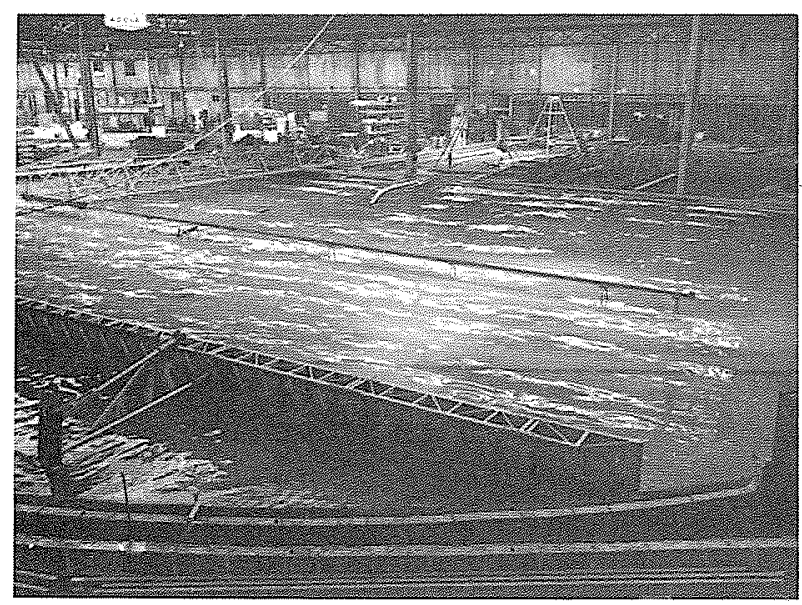

Figure 11 - Cuve à houle.

loi de répartition des amplitudes était conforme à celle des probabilités relevées en nature. De même on a admis que les périodes et les amplitudes étaient reliées par une loi de la forme $T=0,8$ à $1,4 H+7,5$, relation mise en évidence pour les houles du Golfe de Gascogne.

La marée était schématisée pour représenter une loi sinusoïdale de $3,65 \mathrm{~m}$ d'amplitude correspondant à une marée de vives-eaux du Golfe de Gascogne et allant de la cote $+4,30 \mathrm{~m}$ à la cote $+0,65 \mathrm{~m}$, le niveau moyen étant à la cote $2,45 \mathrm{~m}$ environ.

Les fonds étaient supposés en pente douce de 1,5\% en nature. Ils étaient représentés en cuve à houle entre les cotes $+7,50 \mathrm{~m}$ et $-40 \mathrm{~m}$ environ.

Les courants généraux et les courants de marée n'étaient pas, par contre, représentés sur le modèle pour cette série d'expériences.

Les sédiments naturels ont été schématisés par des matières plastiques de densité 1,38 avec une répartition granulométrique conforme à celle mesurée en nature, le diamètre moyen des grains artificiels étant de $255 \mathrm{mi}$ crons pour les études en canal et représentant des sables compris entre 60 et 300 microns en nature, et de 375 microns pour les études en cuve à houle représentant un sable de 250 microns de diamètre moyen.

Les souilles draguées avaient, pour les études en canal, un profil trapézoidal, la largeur à la base étant voisine de $80 \mathrm{~m}$ et celle au sommet de $140 \mathrm{~m}$, la profondeur étant de $5 \mathrm{~m}$. Une telle souille représente une section de $550 \mathrm{~m}^{3} / \mathrm{m}$. La souille était réalisée sur toute la largeur du canal.

En cuve à houle, un profil légèrement différent a été adopté afin de se rapprocher des indications fournies par des dragueurs. La souille expérimentale avait en nature une longueur de $800 \mathrm{~m}$ ( $4 \mathrm{~m}$ sur le modèle), une largeur au sommet de $200 \mathrm{~m}$ et une profondeur de $6 \mathrm{~m}$. Les pentes des berges de la souille étaient à 5 pour 1 et le volume correspondait à une extraction de $800000 \mathrm{~m}^{3}$ de matériau $\left(1000 \mathrm{~m}^{3}\right.$ par mètre linéaire).

Précisons que l'échelle des temps sédimentologiques applicable à l'ensemble des études en canal et en cuve à houle était de $1 / 1500$, une minute sur le modèle représentant environ un jour en nature.

De même rappelons que les amplitudes indiquées correspondent au $H_{1 / 10}$ et que les amplitudes significatives $H_{1 / 3}$ sont 1,2 fois plus faibles et les amplitudes moyennes 1,95 fois plus petites.

\section{Etudes en canal vitré}

Les fosses de dragages en canal vitré étaient supposées draguées dans les fonds de $-7 \mathrm{~m},-12 \mathrm{~m},-15 \mathrm{~m}$ et $-20 \mathrm{~m}$, ces valeurs étant données par rapport au zéro hydrographique correspondant aux plus basses-mers de vives-eaux exceptionnelles.

Ces souilles ont été soumises à des houles d'amplitudes croissantes de $1,90 \mathrm{~m}, 2,5 \mathrm{~m}, 2,85 \mathrm{~m}, 3,15 \mathrm{~m}$, $4,40 \mathrm{~m}, 5,0 \mathrm{~m}, 5,65 \mathrm{~m}, 6,25 \mathrm{~m}, 6,90 \mathrm{~m}$ et $7,50 \mathrm{~m}$ et l'on observait leur évolution en fonction du temps d'action des houles. Chaque type de houle agissait pendant une durée équivalente à 32 jours en nature.

Le comblement de ces souilles de dragages est fonction de la profondeur et des caractéristiques de la houle mais, si il existe une relation entre ces paramètres, le processus de comblement reste toujours le même (fig. 12) :

- tant que l'amplitude critique de la houle n'est pas atteinte la fosse est très peut perturbée, le fond ne se comble pas et seuls quelques "coups de griffes" apparaissent,

- lorsque l'on augmente l'amplitude de la houle les coups de griffes se transforment en petites excavations d'où partent des cônes d'épandage avec début d'écoulement des sables au fond de la souille. L'importance du comblement reste toutefois négligeable et il faut une houle encore plus forte pour que les cônes d'épandage atteignent le plafond de la souille. L'érosion côté terre est supérieure à l'érosion côté mer,

- dans un nouveau stade, des rides apparaissent sur les flancs de la souille et gagnent progressivement le centre de la fosse. En augmentant l'amplitude des houles les phénomènes s'intensifient et le fond de la fosse se comble avec apparition de rides d'oscillation,

- progressivement les pentes de la fosse s'adoucissent et se raccordent aux fonds avoisinants qui s'érodent pour venir alimenter la souille qui ne présente plus une individualité propre et s'intègre au profil général. Après un temps d'action assez long la fosse est 


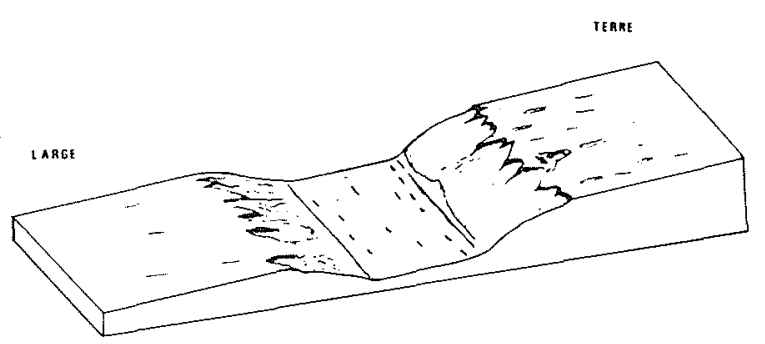

IDébut dërasion

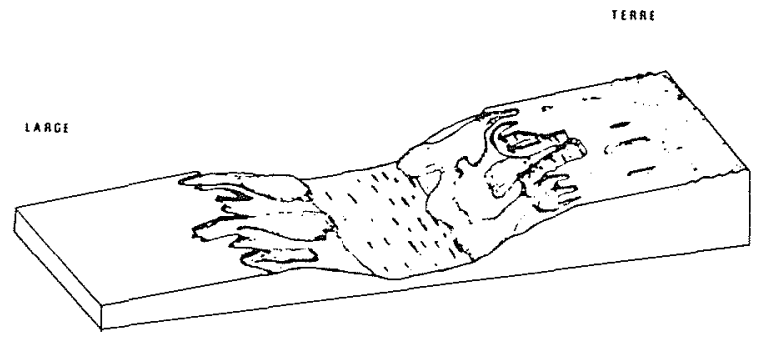

2. Eboulement des talus

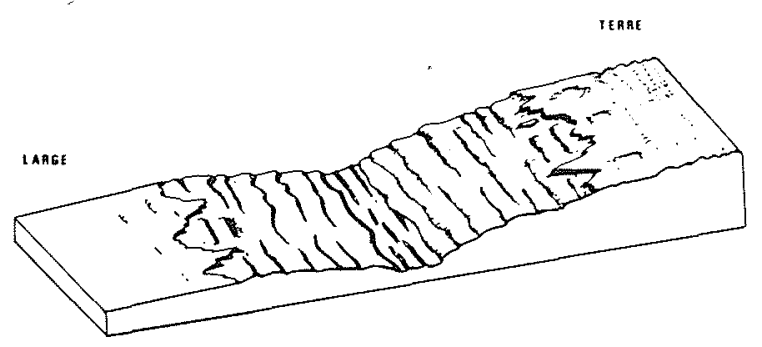

(3) Erosion latérale des fonds

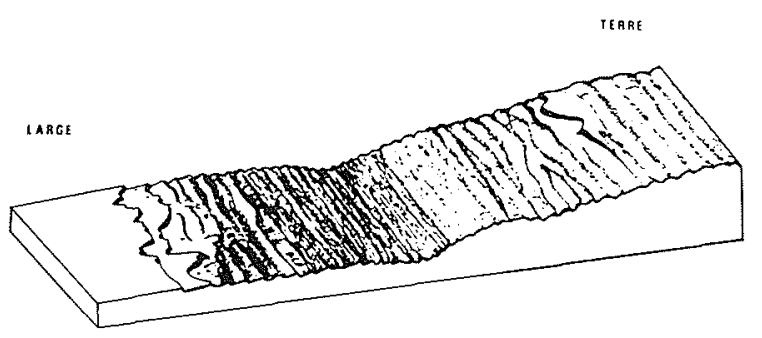

(4) Comblement avec rides

Figure 12 - Processus de comblement d'une souille de dragage.

comblée, la plus grosse érosion apparaissant du côté terre (fig. 13).

En rappelant que la souille expérimentale a une profondeur de $5 \mathrm{~m}$ sous le niveau initial des fonds, son comblement pour différentes houles peut être résumé dans le tableau ci-après et sur la figure 14 . Ces valeurs de comblement correspondent à une durée d'action de la houle de un mois en nature, les fonds ayant pris leur état d'équilibre après cette période.

\begin{tabular}{|l|l|l|l|l|l|l|l|l|l|l|}
\hline \multicolumn{7}{|c|}{ Epaisseur des dépôts (en my dans l'axe de la souille } \\
aprês 1 moi is $d$ d'action des houles \\
\hline Amplitude $H_{1} / 10$ en m & 1,90 & 2,50 & 3,75 & 4,40 & 5,00 & 5,65 & 6,25 & 6,90 & 7,50 \\
\hline Fosse à -7 m & 0 & 0,15 & 0,50 & 2,00 & 2,25 & 2,65 & - & - & - \\
\hline Fosse à -12 m & 0 & 0 & 0,80 & 2,60 & 3,50 & 3,80 & - & - & - \\
\hline Fosse à -15 m & 0 & 0 & 0 & 0,30 & 1,50 & 2,50 & 3,00 & - & - \\
\hline Fosse à -20 m & 0 & 0 & 0 & 0 & 0 & 1,00 & 2,20 & 3,40 & - \\
\hline
\end{tabular}
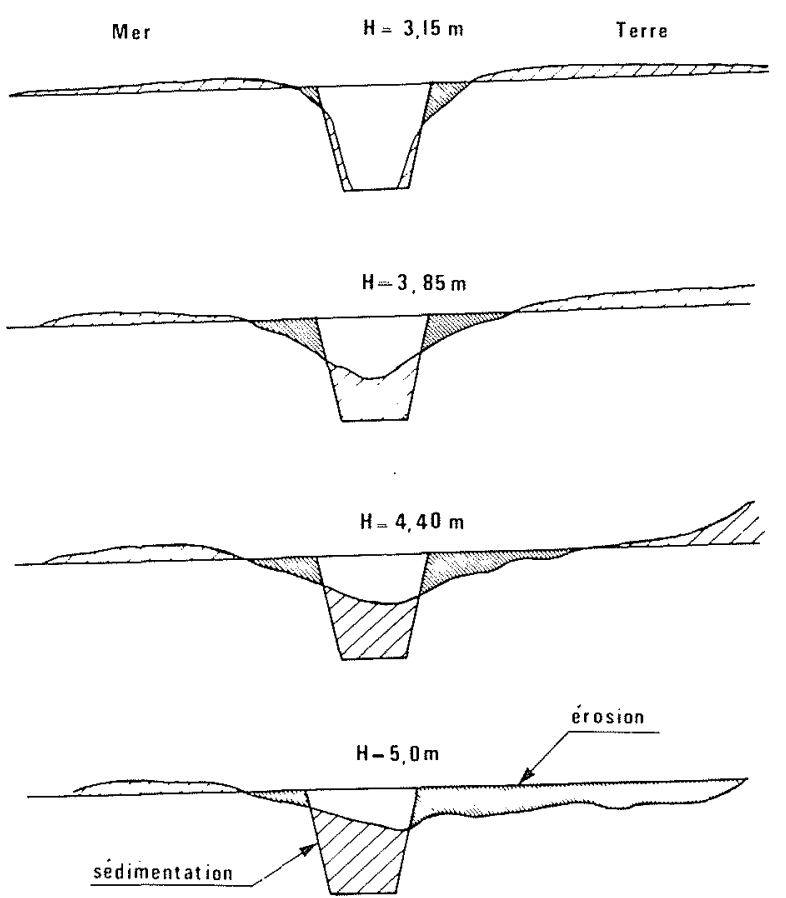

Figure $13-$ Fosse à $-12 \mathrm{~m}$. Exemple d'évolution des fonds en canal vitré après une période de 1 mois d'action de houle de différentes amplitudes.
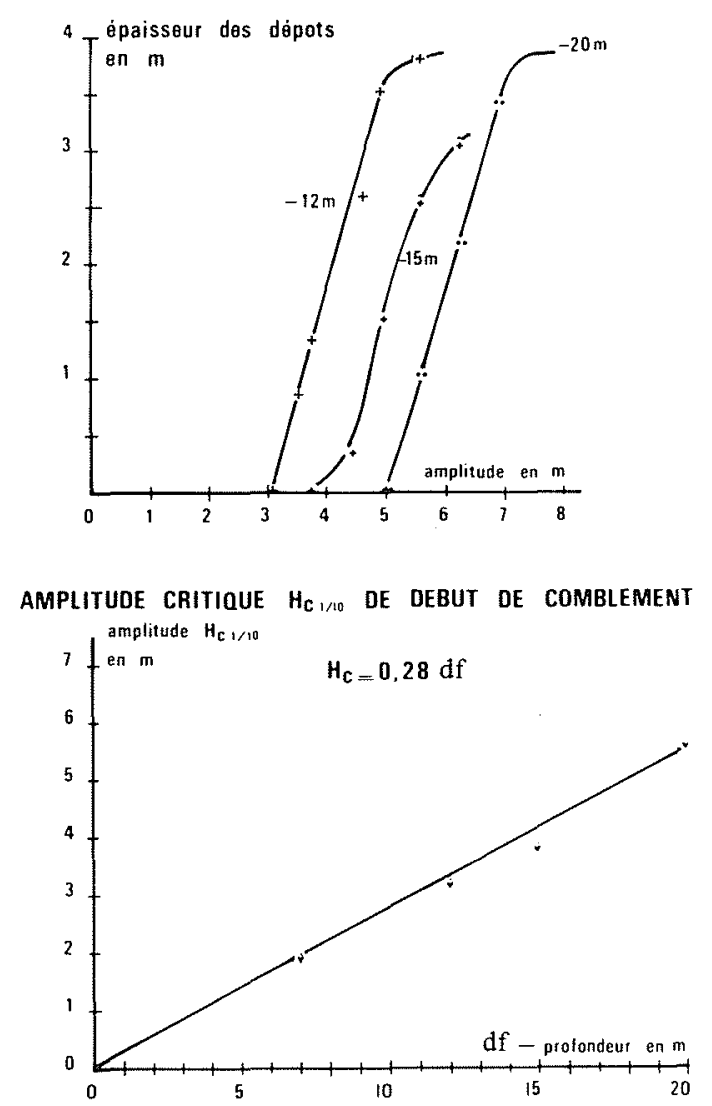

Figure 14 -Comblement en fonction de l'amplitude de la houle. 
D'après ces résultats, on constate que le début de comblement d'une souille draguée à une profondeur " $d f$ " sous le zéro hydrographique, s'effectue à partir d'une houle d'amplitude critique $H c$ donnée par l'expression simplifiée:

$$
H c=0,25 d f
$$

En canal, les essais étant effectués à deux dimensions, on constate que le comblement de la souille s'effectue simultanément à une érosion des fonds situés de part et d'autre avec prédominance de l'érosion côté terre. Les épaisseurs de sédiment " $e$ " déposés dans la souille après un temps constant d'action d'une houle d'amplitude déterminée, correspondant à un mois en nature, augmentent proportionnellement à l'amplitude efficace de la houle $\mathrm{He}$ déterminée comme étant la différence entre l'amplitude mesurée $H$ et l'amplitude critique $H_{c}$

$$
e=K(H-H c)^{1,5}
$$

Il s'agit là d'un ordre de grandeur des phénomènes, cette expression n'étant dans tous les cas applicable que pour les souilles situées au large du déferlement et les valeurs des dépôts tendant vers une valeur asymptotique lorsque le comblement s'effectue. Dans tous les cas le fait de réaliser les études en canal à deux dimensions, ne permet d'avoir qu'une vue approchée des phénomènes ; les résultats obtenus recoupent cependant assez bien ceux qui avaient été donnés à partir de considérations théoriques ou de mesures en nature à l'aide de traceurs radioactifs.

Etude en cuve à houle de l'évolution des différentes fosses soumises à l'action cumulative des houles d'amplitude croissante agissant pendant une courte durée.

En introduisant la troisième dimension, la cuve à houle construite à la demande du C.N.E.X.O. offre un outil de travail beaucoup plus performant pour examiner le comportement de souilles draguées à différentes profondeurs. Les courants de houle, de retour, d'expansion latérale, les rip-currents peuvent se reproduire automatiquement lorsque les fonds sont attaqués par les houles. Il est possible également de faire varier en continu non seulement les amplitudes et périodes mais également les directions et de programmer un "cycle annuel" représentant très exactement les fluctuations des houles observées en nature.

Sur cette vaste cuve longue de plus de $30 \mathrm{~m}$ et d'une superficie de $600 \mathrm{~m}^{2}$ les souilles expérimentales d'une capacité de $800000 \mathrm{~m}^{3}$ (longueur $800 \mathrm{~m}$, largeur $200 \mathrm{~m}$, profondeur $6 \mathrm{~m}$ ) ont été implantées par des profondeurs de $-6 \mathrm{~m},-11 \mathrm{~m},-16 \mathrm{~m},-21 \mathrm{~m}$ et $-23 \mathrm{~m}$ (fig. 15).

Dans une première partie, ces fosses ont été soumises à des actions de houles frontales de plus en plus fortes et agissant pendant des durées déduites des statistiques des houles dans le Golfe de Gascogne.

Pour les fosses à -11 et $-21 \mathrm{~m}$ on a dans une deuxième partie examiné l'influence des houles obliques dont les crêtes au large faisaient un angle de $15^{\circ}$ avec le littoral.

Ces essais étaient précédés par un "état de référence" réalisé sur les fonds naturels sans fosse de dragage.

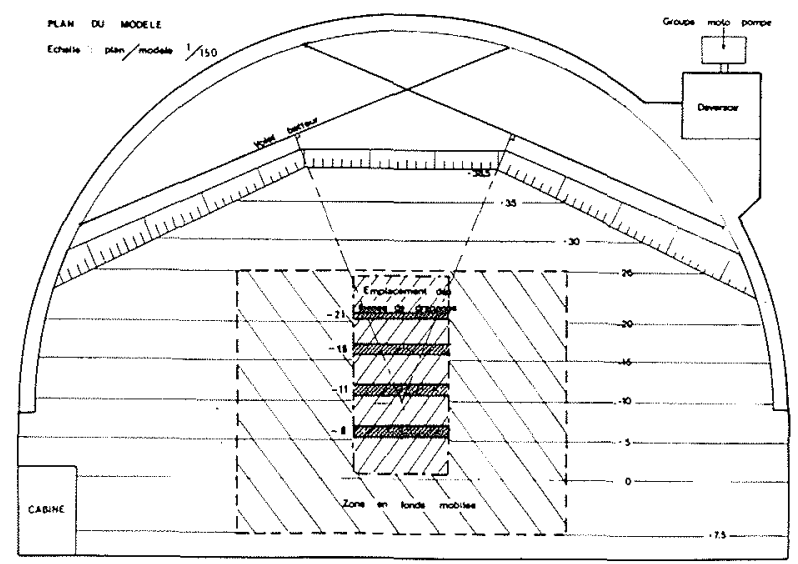

Figure 15 - Répartition des fosses de dragages dans la cuve à houle.

Le cycle élémentaire introduit sur le modèle est représenté dans le tableau ci-après, les amplitudes étant croissantes au cours du temps. Au total deux cycles ont été réalisés représentant des actions cumulatives de la houle

\begin{tabular}{|c|c|c|}
\hline $\begin{array}{c}\text { Amplitude } \mathrm{H}_{1 / 10} \\
\text { (mètres) }\end{array}$ & Période (secondes) & $\begin{array}{l}\text { Duree d'action } \\
\text { (jours) }\end{array}$ \\
\hline 1 a 3 & $\begin{array}{r}8 \\
10 \\
12\end{array}$ & $\begin{array}{l}75 \\
75 \\
75\end{array}$ \\
\hline 3 d 5 & $\begin{array}{l}10 \\
12 \\
14\end{array}$ & $\begin{array}{l}15 \\
15 \\
15\end{array}$ \\
\hline $\begin{array}{lll}5 & \mathrm{à} & 7\end{array}$ & $\begin{array}{l}12 \\
14\end{array}$ & $\begin{array}{l}15 \\
15\end{array}$ \\
\hline
\end{tabular}
pendant 2 années environ.

Cette étude sera complétée en examinant le comportement d'une souille à $-21 \mathrm{~m}$ sous l'action des cycles complexes de houle se rapprochant des observations faites en nature dans le Golfe de Gascogne et agissant pendant 7 années sur les fonds avec des amplitudes pouvant atteindre $13 \mathrm{~m}$.

Comportement des différentes souilles soumises $\grave{a}$ l'action de houles frontales d'amplitude croissante agissant pendant une courte durée

\section{a) Fosse par fonds de $-6 m$}

Cette fosse se situe à proximité du littoral à une distance de $400 \mathrm{~m}$ de l'isobathe 0 et à $900 \mathrm{~m}$ du haut de plage $(+7,50 \mathrm{~m})$.

Les houles de 1 à $3 \mathrm{~m}$ d'amplitude $H_{1 / 10}$, correspondant à des amplitudes moyennes de 0,5 à $1,5 \mathrm{~m}$, ont peu d'influence sur la géométrie de la fosse, et les dépôts, après l'équivalent de 7,5 mois d'action, ne représentent que $56 \mathrm{~m}^{3} / \mathrm{m}$ pour une fosse ayant une section initiale de $1000 \mathrm{~m}^{3} / \mathrm{m}$. Les houles de 3 à $5 \mathrm{~m}$ agissant pendant 1,5 mois provoquent une sédimentation de l'ordre de $1 \mathrm{~m}$ et des dépôts de $220 \mathrm{~m}^{3} / \mathrm{m}$. Celles de 5 à $7 \mathrm{~m}$ 


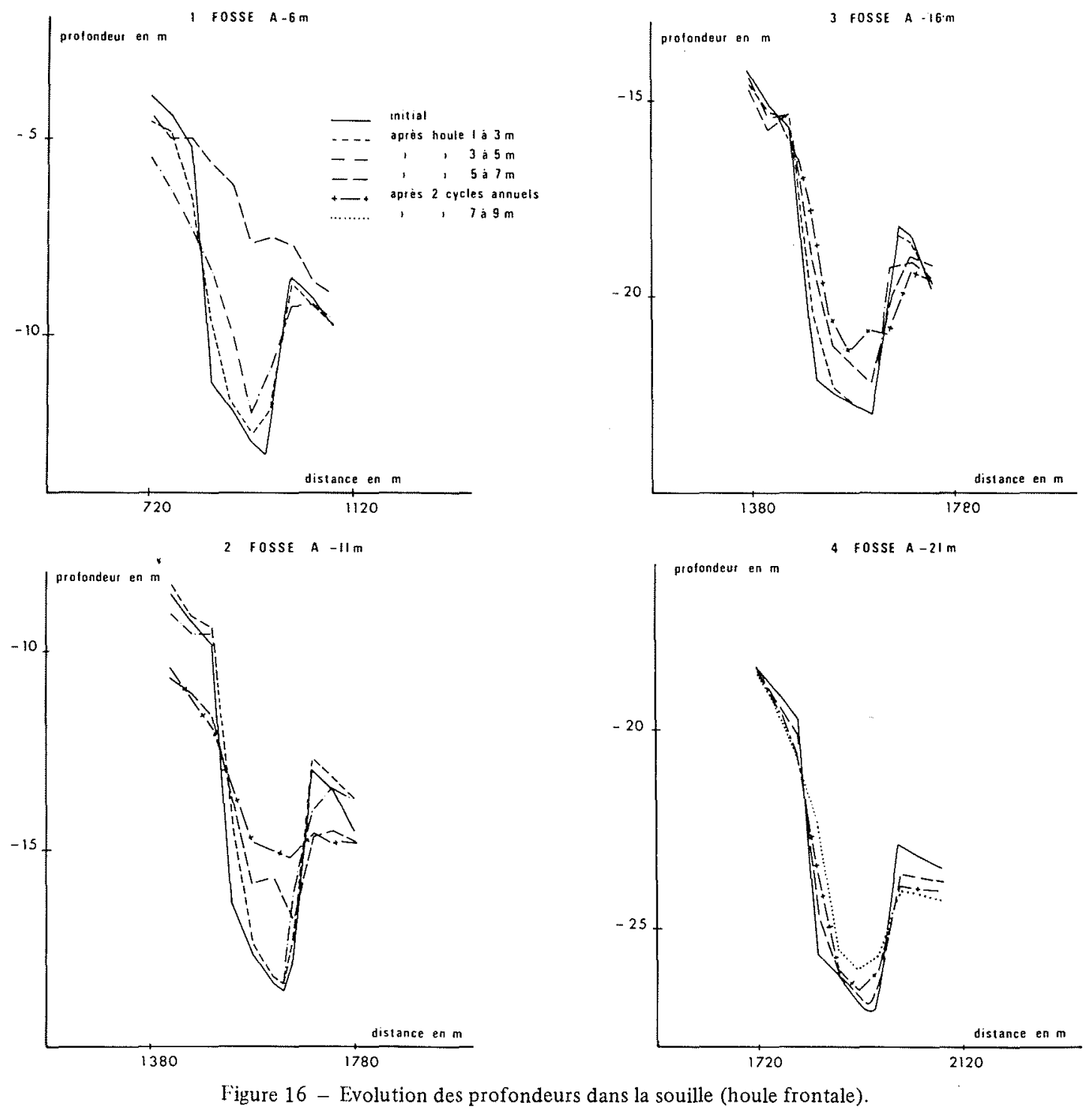

assurent en un mois le comblement presque total de la fosse, les apports atteignant $880 \mathrm{~m}^{3} / \mathrm{m}$ et la hauteur des dépôts 5 à $5,50 \mathrm{~m}$ (fig. 16).

Dès lors la fosse n'est plus apparente, les fonds au niveau de la fosse sont identiques à ceux des fonds avoisinants.

Après l'équivalent d'une année en nature on note une érosion appréciable du littoral qui s'accentue la deuxième année pour atteindre près de $1 \mathrm{~m}$ entre les cotes 0 et $+5 \mathrm{~m}$. Cette érosion est plus marquée dans la partie du littoral située derrière les extrémités de la fosse de dragage que dans l'axe de celle-ci.

\section{b) Fosse par fonds de $-11 \mathrm{~m}$}

Cette fosse est située à $750 \mathrm{~m}$ de l'isobathe 0 et à $1250 \mathrm{~m}$ de l'isobathe $+7,50 \mathrm{~m}$.

Les houles de 1 à $3 \mathrm{~m}$ n'ont aucune influence sur la fosse et les houles de 3 à $5 \mathrm{~m}$ ne provoquent qu'un apport de $80 \mathrm{~m}^{3} / \mathrm{m}$ de sable. Par contre les tempêtes de 5 à $7 \mathrm{~m}$ entraînent dès la fin du $1^{\text {er }}$ cycle $(1$ mois d'action) un dépôt de $320 \mathrm{~m}^{3} / \mathrm{m}$, cette valeur passant à $440 \mathrm{~m}^{3} / \mathrm{m}$ à la fin du $2^{\mathrm{cme}}$ cycle. Ce n'est pratique- ment qu'en période hivernale qu'une telle fosse pourra se combler (fig. 16).

Le comblement de la fosse s'effectue au détriment des fonds situés entre -5 et $-11 \mathrm{~m}$ où l'on constate une érosion moyenne de $2 \mathrm{~m}$ en deux ans. Sur l'estran par contre l'érosion n'est sensible que sur les parties situées en extrémité de la fosse de dragage, dans l'axe de la fosse la côte reste stable ou en légère sédimentation. Ce fait peut être dû à la réfraction de la houle sur la fosse de dragage et les petits fonds érodés et à son épanouissement local dans ce secteur. Sur une plus longue période la côte devrait s'éroder (fig. 17).

\section{c) Fosse par fonds de $-16 \mathrm{~m}$}

La géométrie de la fosse n'est modifiée que par les houles de 5 à $7 \mathrm{~m}$ d'amplitude qui causent une sédimentation de l'ordre du mètre. Après deux ans le volume de sédiment qui s'est déposé dans l'axe de la fosse est de l'ordre de $250 \mathrm{~m}^{3} / \mathrm{m}$, il était de $170 \mathrm{~m}^{3} / \mathrm{m}$ après un an. Au total, compte tenu de la répartition des dépôts dans la souille le volume total des apports atteint 

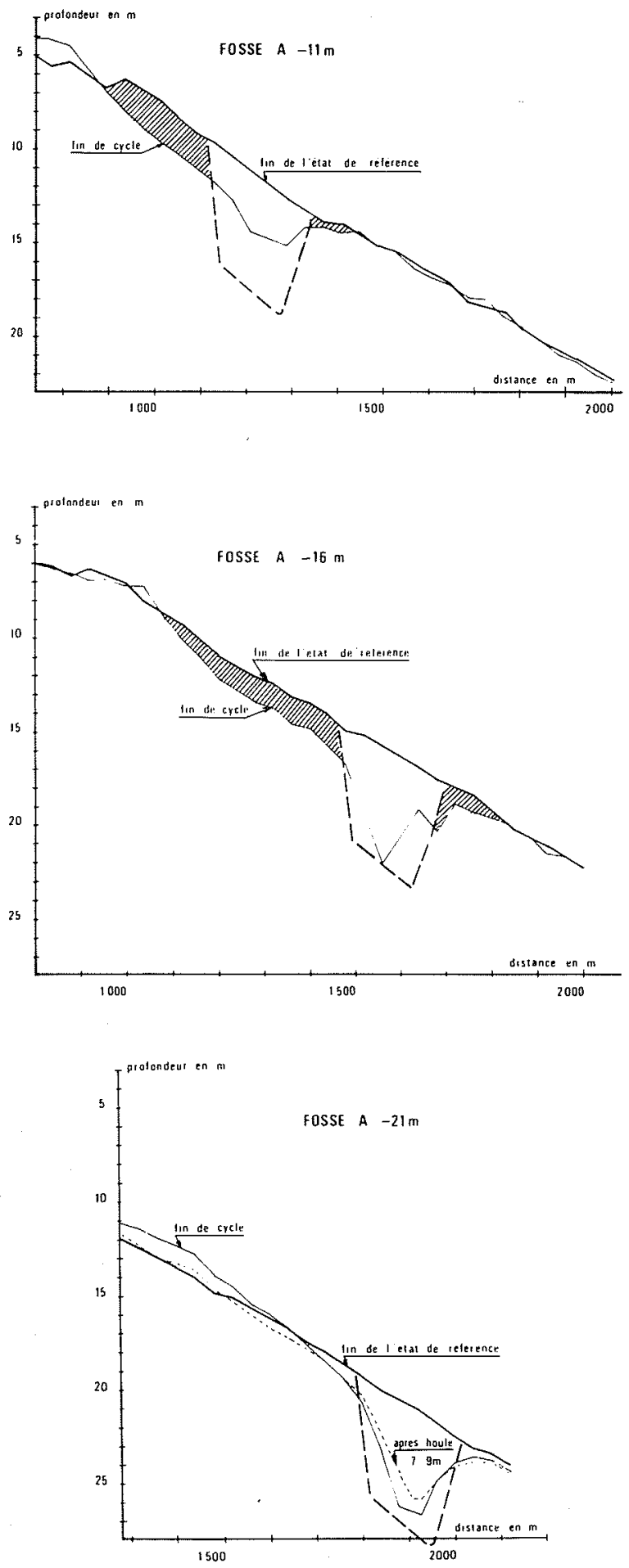

Figure 17 - Evolution des fonds en cuve à houle après 2 ans d'action de houles frontales.

$200000 \mathrm{~m}^{3}$ en deux ans, soit le quart de la capacité de la fosse (fig. 16).

Les petits fonds situés entre les isobathes $-8 \mathrm{~m}$ et $-11 \mathrm{~m}$ se sont érodés, les sédiments venant s'ébouler dans la fosse. Par contre dans l'axe de la fosse l'estran a conservé au cours des deux cycles annuels une certaine stabilité, les érosions n'apparaissant que dans les zones situées en extrémité de la fosse de dragage. d) Fosse par fonds de $-21 \mathrm{~m}$

Cette fosse est située à 1400 mètres de l'isobathe 0 et à 1900 mètres de l'isobathe $+7,50 \mathrm{~m}$.

Les houles inférieures à $7 \mathrm{~m}$ d'amplitude n'ont eu pratiquement aucune action sur la fosse. Il faut atteindre des amplitudes de 7 à $9 \mathrm{~m}$, agissant pendant 15 jours consécutifs, pour que l'on note dans l'axe de la souille un dépôt de $0,75 \mathrm{~m}$ d'épaisseur et que la sédimentation totale atteigne $100000 \mathrm{~m}^{3}$, soit $126 \mathrm{~m}^{3} / \mathrm{m}$. La probabilité de ces houles étant voisine de 1 jour tous les 2 à 5 ans dans le Golfe de Gascogne, les risques de colmatage sont assez réduits (fig. 16).

Après deux années cette fosse n'a pas eu d'influence appréciable sur les fonds littoraux. Seule la zone située à une centaine de mètres autour de la souille a vu ses fonds s'abaisser de $1,00 \mathrm{~m}$ en moyenne, phénomène principalement dû à un adoucissement de la pente des berges de la fosse.

La zone d'influence de cette fosse est très limitée, et il n'y a pas à l'échelon de deux années de risques pour le littoral lorsque les houles sont frontales et ne dépassent pas $7 \mathrm{~m}$ d'amplitude; les effets d'une courte tempête très exceptionnelle de 7 à $9 \mathrm{~m}$ n'apportent pas de dégâts appréciables.

Notons qu'un essai complémentaire effectué en admettant que la fosse de dragage était réalisée le long d'une côte sableuse comportant un haut estran réfléchissant (mur de défense du haut de plage vertical) a montré que le comblement de cette fosse pouvait être sensiblement plus important qu'au cours des essais précédents et que des érosions des petits fonds pourraient en résulter.

Comportement de souilles $\grave{a}-11$ et $-21 \mathrm{~m}$ sous l'action d'une houle oblique agissant pendant une courte période (2 ans nature)

Pour préciser le comportement des souilles de dragage, l'étude précédente, réalisée avec des houles frontales, a été complétée en examinant l'influence d'une houle oblique se présentant au large avec un angle constant de $15^{\circ}$ par rapport à la ligne de rivage .

\section{a) Fosse par fonds de $-11 \mathrm{~m}$}

On retrouve avec les houles obliques des résultats voisins pour le comblement de la souille de ceux que l'on avait avec les houles frontales.

Pour une fosse à $-11 \mathrm{~m}$ les houles de 1 à $3 \mathrm{~m}$ n'ont pas d'influence, celles de 3 à $5 \mathrm{~m}$ provoquant un dépôt de $0,50 \mathrm{~m}$ dans l'axe de la souille et les houles de 5 à $7 \mathrm{~m}$ un dépôt de près de $300 \mathrm{~m}^{3} / \mathrm{m}$ à la fin du $1^{\text {er }}$ cycle annuel.

La différence provient dans la répartition des dépôts qui sont légèrement supérieurs dans la zone attaquée en premier par les crêtes de houles, par suite de la présence de courants de houles. On constate également que la pente de la souille est plus faible du côté large que du côté terre, ce qui était inverse avec les houles frontales (fig. 18).

Entre la souille de dragage et le littoral on constate : 
EVOLUTION DE LA GEOMETRIE DE LA FOSSE A -11,0 m

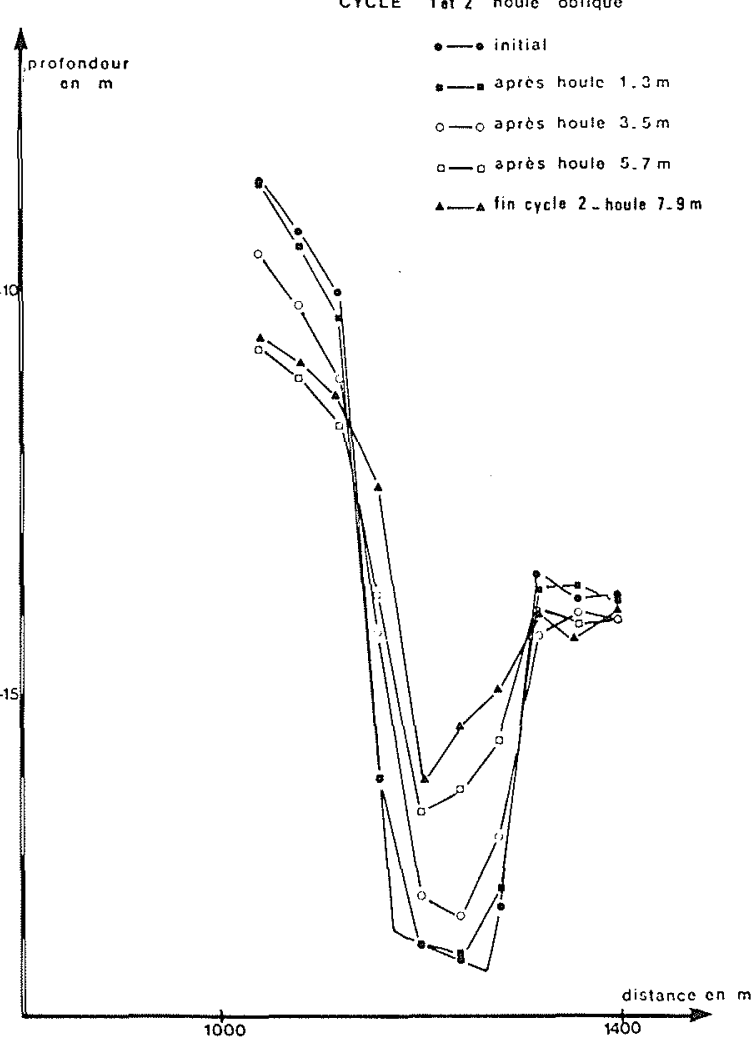

EVOLUTION DE LA GEOMETRIE OE LA FOSSE A $-27.0 \mathrm{~m}$

CYCLE 1 it 2 Houle oblique

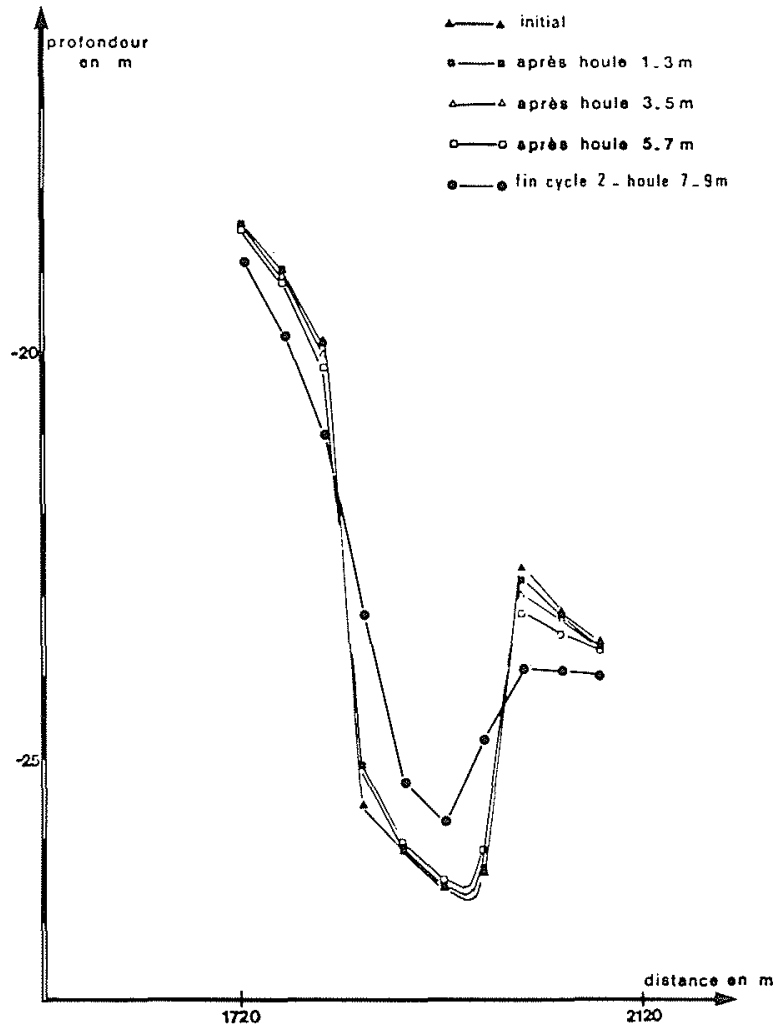

Figure 18 - Evolution des fonds sous l'action de houles obliques.
- peu de modifications des fonds situés au-dessus de l'isobathe $-5,00$. Il n'y a pas en particulier le léger engraissement de l'estran observé derrière la fosse en houles frontales,

- une érosion de $2 \mathrm{~m}$ des fonds situés entre l'isobathe $-7,00$ et la fosse, sur 300 mètres environ. Cette zone érodée est toutefois moins importante qu'en houle frontale.

Après deux cycles annuels on n'est pas arrivé à un état d'équilibre et comme pour la fosse étudiée en houle frontale il est certain que le littoral sera à terme menacé.

\section{b) Fosse par fonds de $-21 \mathrm{~m}$}

Seules les houles de 7 à 9 mètres modifient sensiblement la géométrie de la fosse. Après deux années il s'y est déposé $75000 \mathrm{~m}^{3}$, ce qui représente environ $45 \mathrm{~m}^{3}$ / $m$ et par an. L'équivalent de 15 jours de houles obliques de 7 à $9 \mathrm{~m}$ d'amplitude apporte $100000 \mathrm{~m}^{3}$ de sable.

Après deux ans on n'observe pas une évolution sensible des fonds littoraux en face de la fosse. Ce résultat est identique à celui obtenu en houle frontale mais il aurait été souhaitable d'avoir des renseignements plus précis sur l'évolution du littoral très en aval de la fosse pour examiner si le transit littoral pourrait être, à la longue, perturbé.

Comparaison du comblement des différentes fosses de dragage soumises à l'action des houles frontales et obliques

Partant des évolutions des fonds constatées au cours des différents essais, des lois permettant d'évaluer les risques de colmatage des différentes fosses de dragage ont été recherchées.

a) Rehaussement du centre de la fosse, amplitude critique de comblement

Le tableau ci-après donne le rehaussement moyen du centre de chaque fosse de dragage $(6 \mathrm{~m}$ de profondeur initiale) pour chaque classe d'amplitude de houle.

\begin{tabular}{|c|c|c|c|c|c|c|}
\hline \multicolumn{7}{|c|}{ Rehaussement du centre de la fosse (en m) } \\
\hline & \multicolumn{4}{|c|}{ Houle frontale } & \multicolumn{2}{|c|}{ Houle oblique } \\
\hline \multirow{2}{*}{\begin{tabular}{|c|} 
Fosse \\
Amplitude \\
houle (m)
\end{tabular}} & $-6 \pi$ & $-11 m$ & $16 \mathrm{~m}$ & $-21 \mathrm{~m}$ & $-11 m$ & $-21 m$ \\
\hline & \multirow[b]{2}{*}{0,30} & \multirow[b]{2}{*}{0,13} & \multirow[b]{2}{*}{0,02} & \multirow[b]{2}{*}{0} & \multirow[b]{2}{*}{0,12} & \multirow[b]{2}{*}{0} \\
\hline 1 à 3 & & & & & & \\
\hline 3 a 5 & 1,05 & 0,36 & 0,04 & 0 & 0,39 & 0 \\
\hline 5 à 7 & 4,60 & 1,76 & 0,89 & 0,21 & 1,51 & 0,20 \\
\hline 1 à 3 & - & 0,09 & 0,09 & 0 & 0,06 & 0 \\
\hline 3 à 5 & - & 0,23 & 0,02 & 0 & 0,12 & 0 \\
\hline 5 à 7 & - & 0,94 & 0,22 & 0,09 & 0,97 & 0,16 \\
\hline 7 à 9 & - & - & - & 1,05 & - & 0,69 \\
\hline $\begin{array}{l}\text { Total cumulé } \\
\text { (houle de I à } \\
\frac{7 \mathrm{~m})}{}\end{array}$ & 5,95 & 3,51 & $1,2: 8$ & 0,30 & 3,17 & 0,36 \\
\hline
\end{tabular}




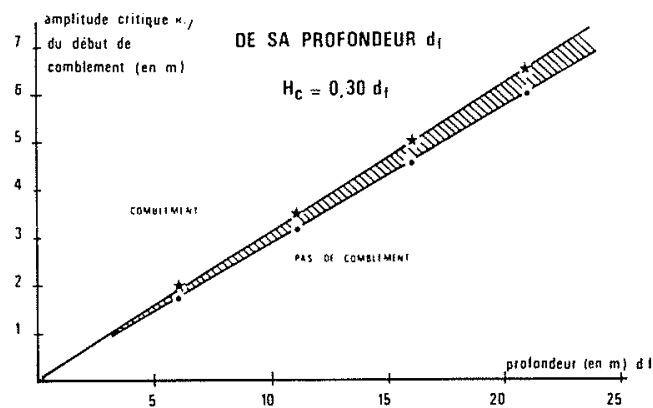

VOLUME DEPOSE DANS UNE SOUILLE DE $800000 \mathrm{~m}^{\prime}$ AU COURS D'UN CYCLE ANNUEL

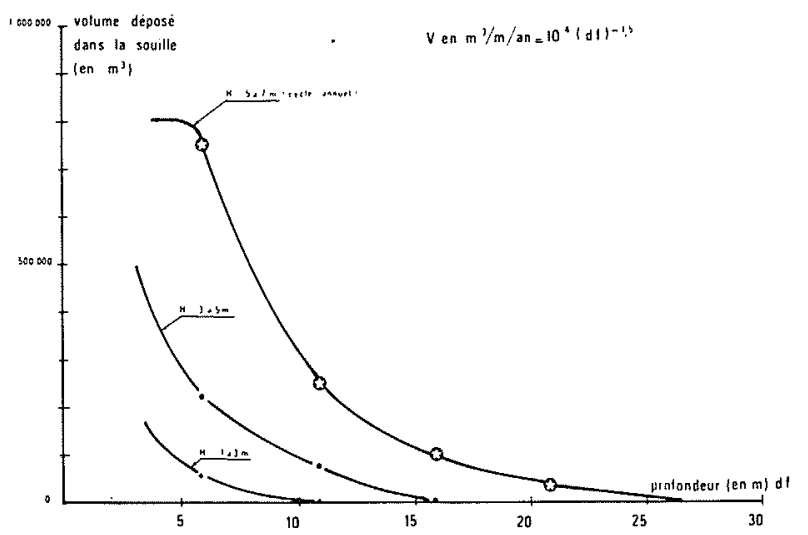

Figure 19 - Amplitude critique $H_{c}$ de comblement d'une souille en fonction de la profondeur $d f$.

Si la fosse à $-6 \mathrm{~m}$ est très rapidement comblée, on remarque que l'épaisseur " $e$ " des dépôts diminue très rapidement avec la profondeur ; par ailleurs, elle est toujours plus faible au cours du deuxième cycle de houle qu'au cours du premier par suite des modifications des talus de raccordement des fosses au cours du temps. L'obliquité de la houle ne semble avoir qu'une faible influence sur le comblement.

En prenant comme limite de début de comblement un rehaussement de 0,10 à $0,20 \mathrm{~m}$ par an, on peut fixer pour chaque profondeur d'implantation de souille " $d f$ " une amplitude critique $H c$ de houle au-delà de laquelle la fosse peut être soumise à un comblement. Cette amplitude critique ( $H_{1 / 10}$ critique) est donnée par la même expression simplifiée que pour les études en canal (fig. 19) :

$$
H c=0,25 \text { à } 0,30 d f
$$

b) Evaluation de l'épaisseur de comblement des fosses en fonction de leur profondeur et des caractéristiques des houles

Connaissant la durée d'action de chaque type de houle, le rehaussement moyen journalier a pu être déterminé et une loi proposée en fonction de l'amplitude "efficace" de la houle caractérisée comme étant la différence entre l'amplitude maximum $H_{M}$ de la classe de houle considérée (par exemple $3 \mathrm{~m}$ pour les amplitudes de la classe 1 à $3 \mathrm{~m}$ ) et l'amplitude critique $H c$ précédemment définie.

La figure 20 donne le rehaussement moyen journalier " $e$ " du centre de la fosse de dragage en fonction de

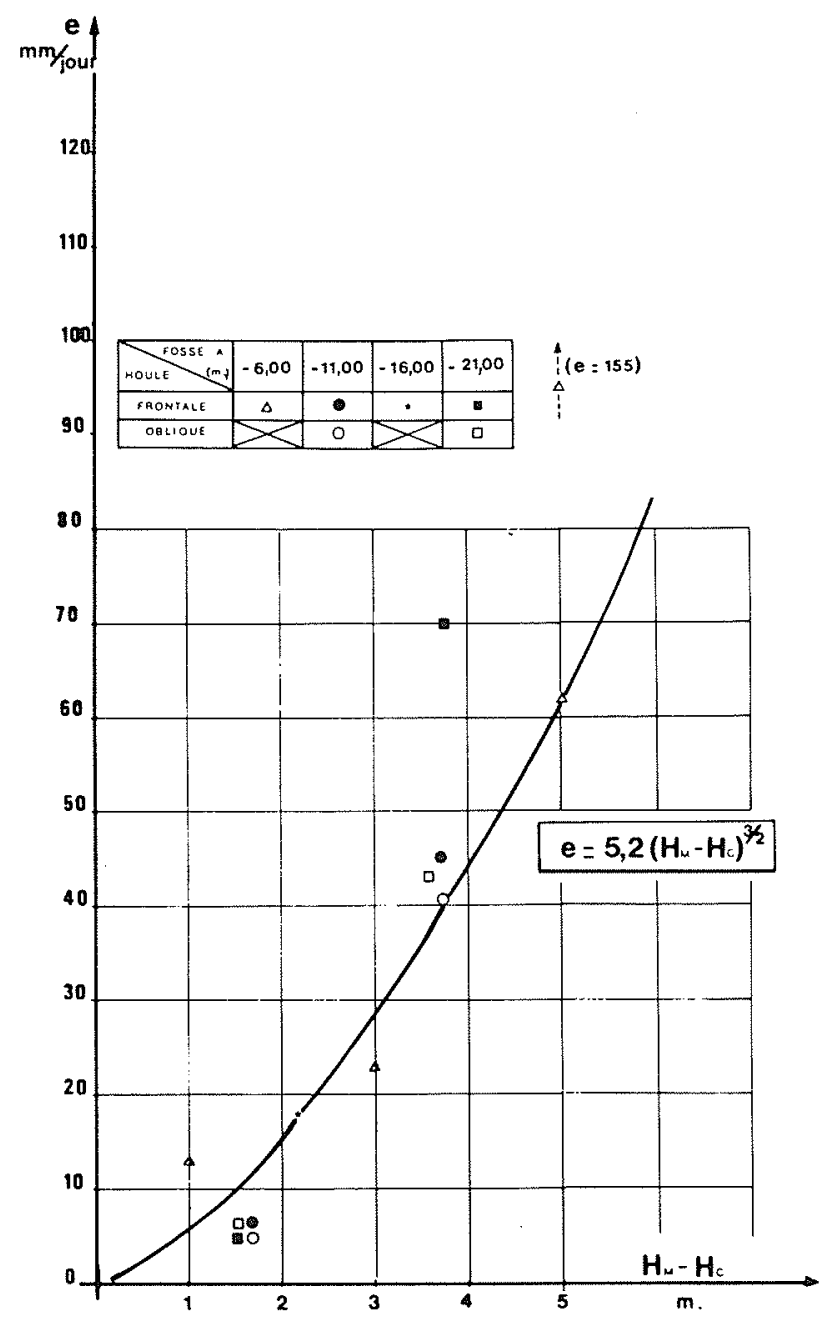

Figure 20 - Rehaussement moyen journalier du centre de la souille en fonction de l'amplitude "efficace" de la houle.

l'amplitude efficace

$$
e(\text { en } \mathrm{mm})=5,2\left(H_{M}-H c\right)^{1,5}
$$

Cette loi est valable pour l'ensemble des souilles situées au large de la zone de déferlement. Pour la souille à $-6 \mathrm{~m}$ sous le zéro des basses-mers on constate que le comblement est beaucoup plus important lorsque les houles de 5 à $7 \mathrm{~m}$ déferlent au voisinage de la fosse.

\section{c) Evaluation des volumes de dépôts dans les diffé-} rentes fosses de dragage

En cubant les dépôts dans les différentes souilles après chaque action des différentes houles et à la fin de chaque cycle annuel, on peut apprécier l'importance des apports.

La figure 21 donne en fonction des différentes profondeurs de fosses les volumes déposés sur la totalité de la fosse à la fin du premier cycle, équivalent à une action cumulative des houles du type Golfe de Gascogne pendant un an, et à la fin du deuxième cycle, correspondant à 2 années nature.

Les volumes des dépôts diminuent rapidement avec la profondeur, et si à la fin du $1^{\mathrm{er}}$ cycle la fosse à $-6 \mathrm{~m}$ s'est totalement comblée, le colmatage de la fosse à 


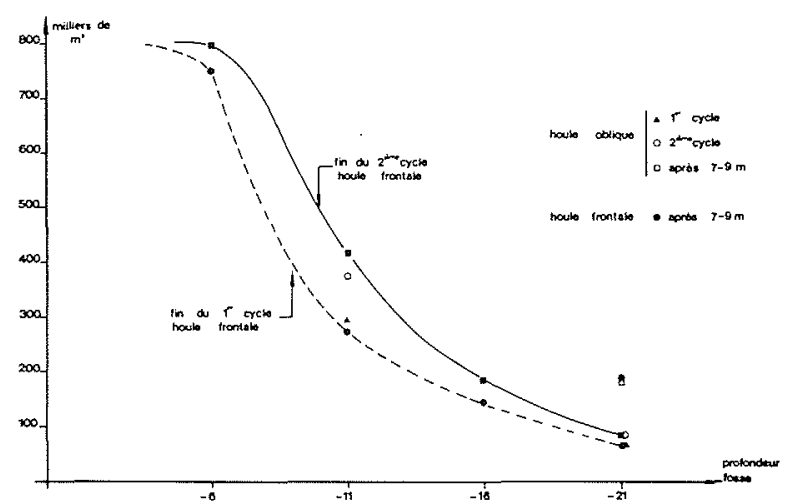

Figure 21 - Dépôts dans les souilles en fonction de leur profondeur.

$-16 \mathrm{~m}$ n'atteint que $25 \%$ à la fin du deuxième cycle, cette valeur tombant à moins de $10 \%$ pour la fosse à $-21 \mathrm{~m}$.

Le tableau ci-après résume les résultats de cette étude du comblement d'une souille de dragage sous l'action des houles frontales de différentes amplitudes, les dépôts étant mesurés dans la partie centrale de la fosse de dragage.

\begin{tabular}{|c|c|c|c|c|c|}
\hline \multicolumn{6}{|c|}{ Volumes cumulés des dépôts dans une souille en $\mathrm{m}^{3} / \mathrm{m}$} \\
\hline $\begin{array}{l}\text { Amp } 1 \text { itude } \\
\text { (en m) }\end{array}$ & $\begin{array}{c}\text { Durée d'action } \\
\text { (en mois) }\end{array}$ & $\begin{array}{l}\text { Prof } \\
-6 \mathrm{~m}\end{array}$ & $\begin{array}{l}s \text { de la } \\
5 \text { basse } \\
-11 \mathrm{~m}\end{array}$ & $\begin{array}{l}\text { Tle sou } \\
\text {-s en m } \\
-16 \mathrm{~m}\end{array}$ & $-21 \mathrm{~m}$ \\
\hline 1 वे 3 & 7,5 & 56 & 0 & 0 & 0 \\
\hline 3 à 5 & 1,5 & 220 & 80 & 0 & 0 \\
\hline 5 a 7 & 1,0 & 880 & 320 & 170 & 30 \\
\hline 7 a 9 & 0,5 & - & - & 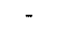 & 125 \\
\hline
\end{tabular}

Sur une année complète, les dépôts par mètre linéaire de souille seraient donnés, dans le cas du Golfe de Gascogne et pour des profondeurs comprises entre -6 et $-21 \mathrm{~m}$, par l'expression :

$$
V \mathrm{~m}^{3} / \mathrm{m} / \mathrm{an}=10^{4} d f^{-1,5}
$$

Le volume journalier des dépôts serait donné en fonction de l'amplitude $H_{M}$ précédemment définie et de l'amplitude critique de début de comblement $H c$ par la relation approchée

$$
V \mathrm{~m}^{3} / \mathrm{m} / \text { jour }=\left(H_{M}-H c\right)^{1,5}
$$

d) Comparaison des dépôts dans les fosses de dragages et des volumes théoriques remaniés par la houle à une même profondeur

En comparant les volumes de dépôts dans les fosses de dragages, pour différentes profondeurs et différentes houles, aux volumes théoriques des sédiments remaniés pour les mêmes houles aux mêmes profondeurs, on constate que la répartition des dépôts suit rigoureusement la même loi à un coefficient multiplicateur près qui est de 2,5 si l'on utilise la formule déduite des équations d'Einstein.

Autrement dit, il se déposerait, dans les conditions expérimentales adoptées, 2,5 fois plus de sédiments dans une fosse de dragage que ne donnerait l'évaluation

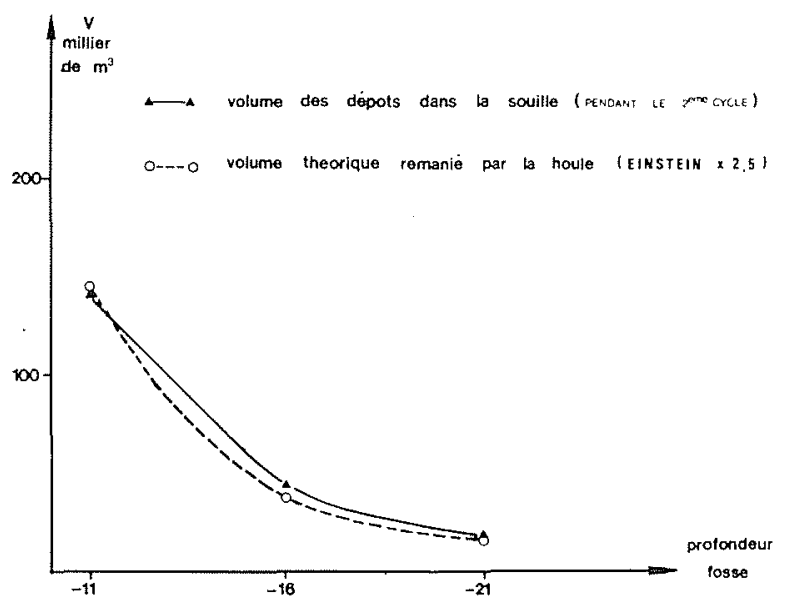

Figure 22 - Comparaison des volumes des dépôts dans les souilles et des volumes théoriques remaniés par la houle.

théorique calculée sur un fond uniforme. La discontinuité des profondeurs et les remaniements des pentes des talus de raccordement suffisent à expliquer ces différences. La figure 22 illustre cette comparaison.

Etude en cuve à houle de l'évolution d'une fosse de dragage à $-21 \mathrm{~m}$ soumise à l'action de cycles de houles identiques à ceux du Golfe de Gascogne et agissant sur une longue période de 7 ans - Influence sur l'équilibre du littoral

Les essais précédents ont permis d'examiner le comportement de fosses de dragages de $800000 \mathrm{~m}^{3}$, implantées successivement par fonds de $-6,-11,-16$ et $-21 \mathrm{~m}$ (sous la cote des plus basses-mers), sous l'action de houles frontales ou d'obliquité constante agissant sur une période n'excédant pas 2 ans.

Pour compléter cette étude et généraliser les résultats à des houles complexes de différentes directions, amplitudes et périodes agissant pendant une longue durée de 7 ans, un essai a été entrepris en examinant le comportement d'une fosse de dragage implantée par fonds de $-21 \mathrm{~m}$ et présentant les mêmes caractéristiques géométriques que les fosses précédentes (profondeur $6 \mathrm{~m}$, largeur au sommet $200 \mathrm{~m}$, pentes de raccordement à 5 pour 1 , capacité $1000 \mathrm{~m}^{3}$ par mètre linéaire). Les fonds étaient supposés constitués de sables de 250 microns en moyenne (150 à 300 microns) et la marée était schématisée entre les cotes $+4,30$ à $+0,65 \mathrm{~m}$.

\section{Caractéristiques des houles reproduites}

Le cycle de houle reproduit au cours de cette étude représentait la succession des différentes caractéristiques de houles relevées en nature dans le Golfe de Gascogne au cours d'une année avec des alternances de tempêtes exceptionnelles durant quelques jours, de houles moyennes, de beau temps... Le cycle annuel était divisé en deux périodes correspondant sensiblement à une période de 6 mois "d'été" et de 6 mois "d'hiver", la répartition des amplitudes, périodes et directions, étant donnée sur la figure 23 . 

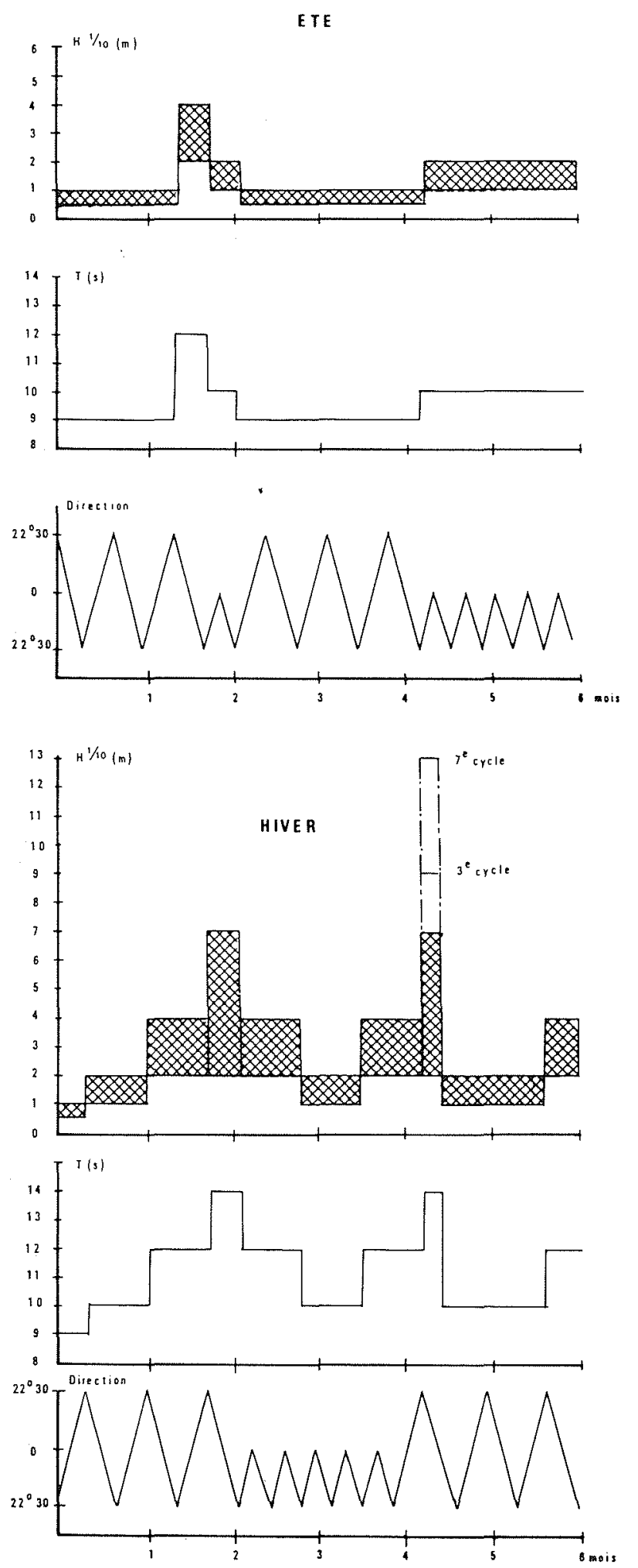

Figure 23 - Cycle de houle reproduit en modèle sur une longue période.

A la fin du $3^{\text {eme }}$ cycle, une tempête de $9 \mathrm{~m}$ d'amplitude agissant pendant l'équivalent de 7 jours en nature était introduite, cette tempête étant portée à $13 \mathrm{~m}$ au cours du $7^{\text {ème }}$ cycle et représentant par conséquent une tempête très exceptionnelle.

Au cours d'un cycle annuel, sous l'action des houles obliques, un transit littoral évalué à $450.000 \mathrm{~m}^{3}$ /an dans un sens et à $250000 \mathrm{~m}^{3} /$ an dans l'autre (transit total cumulé de $700000 \mathrm{~m}^{2} / \mathrm{an}$ ) se produisait, entraínant un transit résultant de $200000 \mathrm{~m}^{3} /$ an.

Evolution des fonds aux abords et à l'intérieur de la fosse de dragage

Pour ce type de souille réalisé par des fonds de $-21 \mathrm{~m}$, la zone d'influence directe de la fosse de dragage reste limitée à son voisinage immédiat, la réper. cussion sur le littoral étant parfaitement inexistante après 7 années comportant des tempetes exceptionnelles.

A l'intérieur de la souille, de légers dépôts apparaissent : ils sont plus importants sur le côté terre de la fosse que sur le côté large et ne dépassent pas $2,50 \mathrm{~m}$ au cours des 5 premières années.

Tant que l'amplitude des houles de tempête ne dépasse pas $9 \mathrm{~m}$, le volume annuel des dépôts atteint en moyenne $45000 \mathrm{~m}^{3}$ par an, c'est-à-dire $5,60 \%$ du dragage initial de $800000 \mathrm{~m}^{3}$. Ce volume de dépôts est légèrement supérieur la première année $\left(70000 \mathrm{~m}^{3}\right)$ et se stabilise ensuite aux environs de $40000 \mathrm{~m}^{3}$ /an (fig. 24).

Avec le cycle comportant des tempêtes exceptionnelles de $13 \mathrm{~m}$ d'amplitude, agissant pendant 7 jours consécutifs, le volume total des dépôts atteint $140000 \mathrm{~m}^{3} /$ an soit $17,5 \%$ de la capacité initiale de la fosse. Il faut préciser que l'existence de ces tempetes exceptionnelles est très rare, la fréquence de retour sur une durée de $24 \mathrm{~h}$ étant supérieure à 10 ans.

La différence de $100000 \mathrm{~m}^{3} /$ an entre ce cycle comportant une tempete exceptionnelle de $13 \mathrm{~m}$ et les cycles précédents est en parfait accord avec les estimations théoriques que l'on avait pu faire à partir des formules données précédemment

$V \mathrm{~m}^{3} / \mathrm{m} /$ jour $=\left(H_{M}-H C\right)^{1,5}$ avec $H c=0,28 d f$

Pour une amplitude $H_{M}$ de $13 \mathrm{~m}$ le volume atteint $19,3 \mathrm{~m}^{3} / \mathrm{m} /$ jour soit $135 \mathrm{~m}^{3} / \mathrm{m}$ pour les 7 jours d'action de la houle et $135 \times 800 \mathrm{~m}=108000 \mathrm{~m}^{3}$ pour la totalité de la souille draguée.

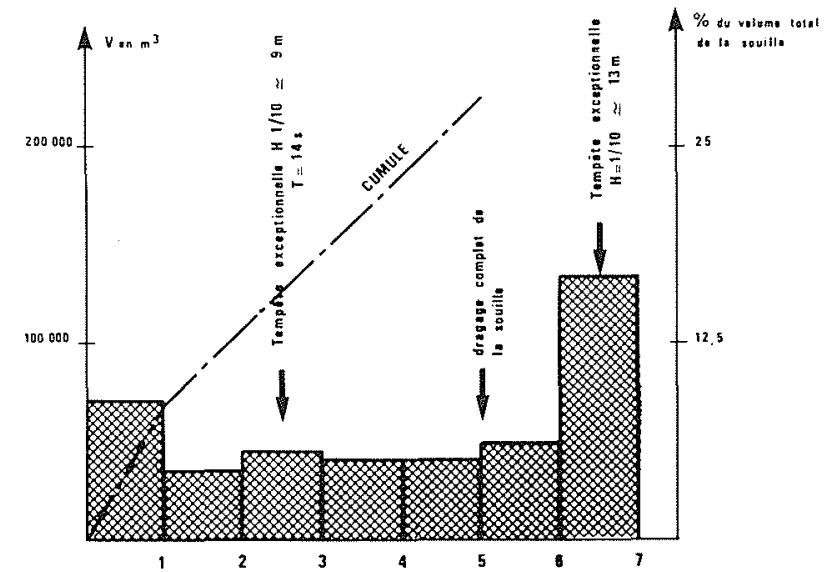

Figure 24 - Variation des volumes des dépôts au cours des 7 cycles de houle. 


\section{Conclusions générales}

L'étude des différentes fosses d'extraction d'agrégats, réalisées parallèlement au rivage par des profondeurs croissantes entre -6 et $-25 \mathrm{~m}$ sous le niveau des plus basses-mers, et soumises à des houles de type "Golfe de Gascogne" permet de formuler les réflexions suivantes:

- Il existe pour chaque profondeur " $d f$ " de souille une amplitudu critique $H c$ de la houle au-delà de laquelle la souille peut être soumise à un comblement. Cette amplitude critique $\left(H_{1 / 10}\right.$ critique) est donnée par l'expression simplifiée :

$$
H c=0,25 \text { à } 0,30 d f
$$

Autrement dit, une souille implantée par fonds de $-20 \mathrm{~m}$ sera stable, à l'échelon de l'année, si les amplitudes $H_{1 / 10}$ ne dépassent pas $6 \mathrm{~m}$ et la période 12 à 14 secondes.

- Les volumes déposés dans les fosses augmentent très rapidement lorsque la profondeur de la fosse diminue et lorsque l'amplitude augmente. Dans le cas de houles frontales ou obliques agissant au large du déferlement, le volume de comblement journalier peut être donné par l'expression très approchée :

$$
V \text { en } \mathrm{m}^{3} / \mathrm{m} / \mathrm{jour}=\left(H_{1 / 10}-H c\right)^{1,5}
$$

Sur une année complète les dépôts par mètre linéaire de fosse seraient donnés, dans le cas du Golfe de Gascogne (bilan d'énergie annuel de $210^{9} \mathrm{~m}^{2} \mathrm{~s}^{2}$ ) et pour des profondeurs de la souille comprises entre -6 et $-35 \mathrm{~m}$ par l'expression:

$$
V \mathrm{~m}^{3} / \mathrm{m} / \mathrm{an}=10^{4} d f^{-1,5}
$$

- Les érosions côté terre de la souille se propagent jusqu'aux fonds de $-5 \mathrm{~m}$ et atteignent des valeurs de $500 \mathrm{~m}^{3}$ par mètre linéaire par les fonds de -11 et -16 mètres. Pour la souille à $-21 \mathrm{~m}$ ces érosions se trouvent limitées au voisinage immédiat de la fosse et ne dépassent pas $80 \mathrm{~m}^{3} / \mathrm{m}$ en deux cycles anmuels.

- Les fosses d'extraction d'agrégats réalisées entre les fonds de -6 et $-11 \mathrm{~m}$ entraînent très rapidement une érosion du littoral, en draguant une fosse par fonds de $-16 \mathrm{~m}$; l'action sur le littoral est beaucoup plus lente, elle devient négligeable à l'échelon de l'année pour une fosse draguée à $-21 \mathrm{~m}$.

- Sur une longue période ( 7 ans) une fosse draguée par fonds de $-21 \mathrm{~m}$, et soumise à des cycles de houle comportant des tempêtes exceptionnelles atteignant $9 \mathrm{~m}, \mathrm{n}$ 'a pas d'influence appréciable sur le littoral, le taux de sédimentation de la fosse ne dépassant pas 5 à $6 \%$ par an de la capacité initiale de la fosse ; ce taux de sédimentation passe à $17 \%$ si l'on effectue des houles de tempêtes exceptionnelles de $13 \mathrm{~m}$ d'amplitude pendant une durée équivalant à 7 jours par an, cette probabilité étant extrêmement rare.

L'ensemble de ces résultats recoupe les valeurs déduites des études théoriques du L.C.H.F., des mesures en nature à l'aide de traceurs radioactifs et des essais en canal vitré.

Pour une souille de caractéristiques identiques à celles étudiées en modèle ( $800 \mathrm{~m}$ de longueur, $200 \mathrm{~m}$ de largeur, $6 \mathrm{~m}$ de profondeur) réalisée dans des fonds sableux en pente douce à $1,5 \%$ et dont la granulométrique moyenne est de 250 microns, on peut estimer qu'il n'y aura pas de répercussions sensibles sur le littoral si cette souille est implantée par des profondeurs supérieures à $-21 \mathrm{~m}$ sous le niveau des plus basses-mers et si les amplitudes $H_{1 / 10}$ des houles restent inférieures à 9 mètres.

Pour généraliser ces résultats et pouvoir donner les autorisations nécessaires aux extractions d'agrégats en mer, il serait souhaitable de compléter ces premières recherches en examinant l'influence éventuelle de courants généraux ou de marées se superposant aux actions des houles et de prendre en considération la forme des souilles de dragages et leurs conditions d'exécution ainsi que la nature des matériaux qui constituent les fonds et la morphologie.

Dans l'état actuel, ces premiers résultats donnent un aperçu des possibilités offertes pour exploiter les ressources sédimentaires marines sans risque appréciable d'entraîner des dégradations irréversibles de notre littoral.

\section{Bibliographie}

[1] BELLESSORT B., LECLERC J.P., MIGNIOT C. --Action de la houle sur les sédiments, Rapport L.C.H.F. Avril 1976, Document C.N.E.X.O.

[2] CAILLOT A. - Etude à l'aide de traceurs radioactifs de l'action de la houle sur les fonds marins, Rapport S.A.R., Octobre 1976.

[3] CHAZAN W. - Une nouvelle source d'approvisionnement : les granulats marins, Annales des Mines, Décembre 1976.

[4] MIGNIOT C. - Action des courants, de la houle et du vent sur les sédiments, L.C.H.F., Houille Blanche, $\mathrm{n}^{\circ} 1$, 1977.

[5] CRESSARD A.P., LEMAIRE J. et LENOBLE J.P. - Les granulats siliceux et calcaires du littoral français. Bulletin liaison Laboratoire Ponts et Chaussées, Spécial IV, Juin 1977.

[6] MARTIN C. - Etude sur modèle réduit physique du comportement de fosses de dragage. Thèse Doctorat troisième cycle, L.C.H.F. et P.M. Curie, Novembre 1977.

[7] CAILlOT A. - Etude de l'action de la houle sur les transports sédimentaires à Sète, Rapport S.A.R., Février 1978.

[8] MIGNIOT C. - Les mouvements sédimentaires sous l'action de la houle, Cours au C.I.F.F., Février 1978.

[9] CRESSARD A.P., GERARD B. - Action du C.N.E.X.O. dans la recherche de granulats marins, Séminaire Germinal, 27 Octobre 1978.

[10] Exposé présenté par le L.C.H.F. au Séminaire sur les ressources minérales sous-marines, B.R.G.M. Germinal, 27 Octobre 1978.

[11] MIGNIOT C. - Utilisation des modèles réduits sédimentologiques pour prévoir l'évolution d'un littoral. Octobre 1978, Congrès Bayonne.

[12] MANOUJIAN S., MIGNIOT C. - Exemple des difficultés de protection du littoral contre l'érosion marine. Octobre 1978 , Congrès Bayonne.

[13] GUIZOL C. - A quel prix commercialiser des granulats marins. Séminaire Germinal, 27 Octobre 1978.

[14] MIGNIOT C. - Problèmes d'érosion et de sédimentation en mer et en rivière, L.C.H.F., En cours publication, Le Moniteur des T.P.

[15] VIGUIER J., MIGNIOT C. - Etude de l'influence des dragages sur l'équilibre des fonds littoraux. Rapport L.C.H.F., Septembre 1979, C.N.E.X.O. D.D.E. Pyrénées Atlantiques, Service Central Technique.

[16] BRIN A. - Les granulats marins, Mission Interministérielle de la mer, 1979. 


\section{Abstract \\ Influence of offshore coarse-grained material extraction on coastal equilibrium}

Sand or gravel extraction from the sea requires respecting precise dredging conditions if damaging conscquences of these extractions on the coastline are to be avoided.

Theoretical studies and ficld measurements yeld the importance of sedimentary movements under wave action at various distances from shore. For the Bay of Biscay, where waves are particularly severe, $20 \mathrm{~m}$ depths under lowest low water level have to be reached to find negligible seasonal sand movements on gently sloping bottoms.

lilume and wave tank studies gave indications on the evolution of sand cxtraction trenches dredged at several depths between shore and $-25 \mathrm{~m}$ bottoms. These trenches were 6 meters deep under sea bottom and $200 \mathrm{~m}$ wide.

Results obtained show that such trenches start filling when the critical wave height $(H c)$ reaches 0.28 times the depth $d f$ at the considered point :

$$
H c=0.25 \text { to } 0.30 d f
$$

The filling rate of these dredging trenches may be determined from the $\mathrm{H} 1 / 10$ wave amplitude by :

$$
V \text { in } \mathrm{m}^{3} / \mathrm{m} / \text { day }=(\mathrm{H} 1 / 10-H c)^{1.5}
$$

Deposits mainly occur by softening of the landward trench slope and generate erosions in shallow bottoms and, consequently, on the beach.

If the dredging trench is further than $-21 \mathrm{~m}$ under low water level, its action on surrounding bottoms and the shoreline is practically inexistant as long as the maximum wave amplitude doesn't go beyond 9 meters.

The results of this study help forecast filling conditions of dredging trenches in sea and to fix limit depths for extracting granulates with respect to local oceanographical conditions so that consequences on the shoreline are negligible.
Génissiat (1950) - $296 \mathrm{p}$

Hydraulique et Electricite Françases (en anglas) 1950. Hydraulique et Electricité Françaises (en anglais) 1950. Bort (1953). - 367 p. . . . . . . . . . . . . . Donzère-Mondragon (1955). $-486 \mathrm{p} . \ldots \ldots \ldots \ldots$ Le Marais Vernier (1957). - 60 p. . . . . . . . . . . Tignes (1958). - 440 p. . . . . . . . . . . . . . . Influence des rejets thermiques sur le milieu vivant en mer et en estuaire. (Journées de la Thermo-Ecologie-Centre Océanologique de Bretagne - Novembre 1976). -622 p. . . .

SOCIETE HYDROTECHNIQUE DE FRANCE / ASSOCIATION INTERNATIONALE DE RECHERCHES HYDRAULIQUES

Recherches sur les turbines hydrauliques (Symposium de Nice, $16-20$ septembre 1960). -431 p. (repr. Xérox) ......
$150 \mathrm{~F}$

$200 \mathrm{~F}$

$175 \mathrm{~F}$

$250 \mathrm{~F}$

$60 \mathrm{~F}$

$210 \mathrm{~F}$

$200 \mathrm{~F}$

$460 \mathrm{~F}$
SOCIETE HYDROTECHNIQUE DE FRANCE: JOURNEES DE L'HYDRAULIQUE

1/ Résumé paru dans La Houille Blanche, n² 27 (B-1949). -

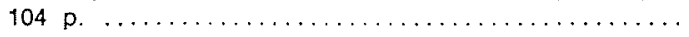

2/ Transport hydraulique et décantation des matériaux solides (1952) . - 224 p. (repr. Xérox) ........ 205 F 3/ Pluie, évaporation, filtration et écoulement (1954). 316 p. (repr. Xérox) .................. $290 \mathrm{~F}$ 4/ Les énergies de la mer (1956). - 752 p........... 575 F 5/ Turbines et pompes hydrauliques (1958). $-730 \mathrm{p} \ldots \ldots 290 \mathrm{~F}$

6/ L'hydraulique souterraine (1960). - 664 p......... $265 \mathrm{~F}$

7/ Bulles et gouttes : la tension superficielle en hydraulique (1962) -624 p. .................... $300 \mathrm{~F}$

$8 /$ Les instabilités en hydraulique et en mécanique des fluides (1964). - $674 \mathrm{p}$. (repr. Xérox) ......... $600 \mathrm{~F}$ 